\title{
Evaluación integral de la política pública de vivienda de interés social en Bogotá, 2008-2016
}

\section{Comprehensive assessment of the public social interest housing policy in Bogota, 2008-2016}

\author{
Mayra Alejandra Lozano Rodríguez \\ Universidad Piloto de Colombia (Colombia) \\ ORCID: http://orcid.org/0000-0001-8780-3882 \\ mayra-lozano@unipiloto.edu.co
}

\section{NOTA BIOGRÁFICA}

Profesional en Relaciones Internacionales y Estudios de la Universidad Militar Nueva Granada (Colombia), Magister en Ciencias Económicas de la Universidad Santo Tomás (Colombia), Estudiante de Doctorado en Estudios Sociales de América Latina de la Universidad Nacional de Córdoba (Argentina). Docente e Investigadora de la Universidad Piloto de Colombia. Áreas de Investigación: Economía Social.

\author{
Hernán Darío Enríquez Sierra \\ Universidad Nacional de Colombia (Colombia) \\ hernan.enriquez@usa.edu.co
}

\section{NOTA BIOGRÁFICA}

Economista y Magister en Economía de la Universidad Nacional de Colombia. Profesor e investigador adscrito a la Escuela de Economía de la Universidad Sergio Arboleda. Miembro del Grupo de Investigación en Políticas públicas y Economía Empresarial y director del semillero de Estudios económicos en migración de la misma Universidad. Experiencia en docencia, investigación y consultoría en áreas de economía regional y urbana, planeamiento urbano y economía de la salud. Áreas de investigación: Economía laboral, Economía Regional y Urbana.

\section{RESUMEN}

El presente documento tiene como objeto evaluar integralmente la efectividad de la política pública de interés social en Bogotá durante el periodo 2008-2016, teniendo como base el planteamiento hecho por Lauchlin Currie, en términos de la correlación existente entre el crecimiento económico y el bienestar, para lograr el desarrollo económico a partir del jalonamiento del sector de la construcción. Se utilizó una metodología que incorpora el análisis de datos macroeconómicos relacionados con el sector de la construcción, la validación del modelo de vulnerabilidad para la asignación de subsidios a través de un modelo probabilístico con el cual se estima la probabilidad de que un hogar acceda a la vivienda, el análisis del comportamiento de condiciones de calidad de vida no inherentes a la ejecución de la política, y la percepción de los hogares objeto, sobre su bienestar.

1 Artículo de revisión producto de la investigación titulada Evaluación Integral De La Política Pública De Vivienda De Interés Social En Bogotá, 2008-2016 para optar el título de Magister en Ciencias Económicas, bajo la dirección de Hernán Enríquez. 


\title{
PALABRAS CLAVE
}

Política pública; vivienda social; interés social.

\begin{abstract}
The purpose of this document is to comprehensively assess the effectiveness of public policy of social interest in Bogotá during the period 2008-2016, based on the approach made by Lauchlin Currie, in terms of the correlation between economic growth and welfare, to achieve economic development from the drawdown of the construction sector. A methodology was used that incorporates the analysis of macroeconomic data related to the construction sector, the validation of the vulnerability model for the allocation of subsidies through a probabilistic model with which the probability of a household accessing the housing, the analysis of the behavior of quality of life conditions not inherent to the execution of the policy, and the perception of the object households, about their well-being.
\end{abstract}

\section{KEYWORDS}

Public policy; social housing; social interest.

\section{SUMARIO}

INTRODUCCIÓN. 1. LA VIVIENDA SOCIAL EN BOGOTÁ. 1.1. ANTECEDENTES. 1.2. LA VIVIENDA COMO MECANISMO PARA EL DESARROLLO ECONÓMICO Y EL MEJORAMIENTO DE LA CALIDAD DE VIDA. 1.3. EVALUACIÓN DE LA POLÍTICA. 1.3.1. Desarrollo Macroeconómico. 1.3.2. Probabilidad de tenencia de vivienda a partir del modelo de vulnerabilidad de la Política. 1.3.3. Análisis de aspectos de calidad de vida no inherentes a la política. 1.3.4. Percepción del nivel de la calidad de vida. 2. RESULTADOS DE LA EVALUACIÓN.

\section{INTRODUCCIÓN}

En el marco de los Derechos Sociales, Económicos y Culturales, la Constitución Política de Colombia (1991), en el Artículo 51 establece que: «Todos los Colombianos tienen derecho a una vivienda digna. El Estado fijará las condiciones necesarias para hacer efectivo este derecho y promoverá planes de vivienda de interés social y sistemas adecuados de financiación a largo plazo».

Con base en lo anterior, el Gobierno Nacional Colombiano ha fijado metas relacionadas con la implementación de programas y estrategias que faciliten la adquisición de vivienda propia, dada la estrecha relación entre la tenencia de vivienda y la pobreza. Es así como la política pública de vivienda de interés social busca privilegiar a los hogares con ingresos menores a 4 SMLV - $\$ 2.757 .820$ (año 2016).

La revisión de diferentes investigaciones realizadas a partir del año 1991 revelan constantes problemas relacionados con el déficit de oferta de vivienda tipo $1^{2}$, el déficit cualitativo de construcciones usadas para vivienda, la asequibilidad a los mecanismos de financiación, y la pobreza inducida, lo que conlleva a plantear el siguiente cuestionamiento ¿La política de vivienda de interés social ha sido efectiva en cuanto al mejoramiento de la calidad de vida de la población en condiciones de pobreza y el desarrollo económico de la ciudad de Bogotá, en el periodo comprendido entre los años 2008 y $2016 ?$

En este sentido, el presente trabajo tiene como objetivo evaluar integralmente ${ }^{3}$ la efectividad de la política pública de interés social en Bogotá a través de: 1 . El análisis de datos macroeconómicos relacionados con el sector de la construcción, 2. la validación del modelo de vulnerabilidad para la asignación de subsidios a través de un modelo «logit», por cuanto es el principal mecanismo de ejecución de la política de vivienda de interés social, 3 . El análisis del comportamiento de condiciones de calidad de vida no inherentes a la ejecución de la política, y 4. La percepción de los hogares objeto.

\footnotetext{
2 Es decir la que su precio no excede el valor de 70 SMLV, también denominada Vivienda de Interés Prioritario.

3 Se menciona un modelo de evaluación integral, por cuanto al hacer la revisión del estado del arte, se identificó que la mayoría de los estudios atienden de forma independiente algunos aspectos de la política de vivienda (cobertura, ejecución presupuestal, calidad infraestructural, entre otros), sin correlacionar las dimensiones mencionadas por Currie desarrollo económico, percepción sobre bienestar y mejoramiento de calidad de vida.
} 


\section{LA VIVIENDA SOCIAL EN BOGOTÁ}

En este apartado se presenta una revisión de las investigaciones previas realizadas en Colombia con relación al problema de la vivienda social y su evaluación, es decir un diagnóstico de los resultados de la ejecución de la política habitacional en el país a partir de los años 90 . Así mismo se presenta el concepto de vivienda social y su evolución teórica; y el concepto de efectividad que será tomado para esta investigación, en el marco de la evaluación de una política pública.

\subsection{Antecedentes}

La pregunta que se trata de responder ¿La política de vivienda de interés social ha sido efectiva en cuanto al mejoramiento de la calidad de vida de la población y el desarrollo económico de la ciudad de Bogotá, en el periodo comprendido entre los años 2008 y 2016 ? implica revisar los estudios previos realizados por otros investigadores y los resultados encontrados, como línea base para identificar los avances en el impacto de la política con relación a escenarios pasados:

Los hallazgos de los diferentes trabajos de evaluación de política pública de vivienda en Colombia se centran principalmente en tres aspectos: el primero tiene que ver con el déficit cuantitativo, es decir la reducida oferta de unidades a precios coherentes con las características económicas de la población objeto. El segundo está relacionado con la incapacidad crediticia de los hogares con ingresos menores a 4 smlv para acceder al crédito de vivienda, o dicho de otra manera los inconvenientes para asumir los requerimientos de las entidades financieras; $y$ el tercero con el desmejoramiento de la calidad de vida de los hogares beneficiarios de la política y el déficit cualitativo.

El déficit cuantitativo es mencionado por Chiappe (1999), quien analizó el desarrollo de la política de vivienda de interés social en Colombia a partir de 1991, cuando se adoptó el sistema de subsidios a la demanda ${ }^{4}$, encontrando que gran parte de los beneficiarios desistieron de utilizarlo debido a que no encontraron predios cuyos precios se adecuaran a su nivel de ingreso, es decir la oferta de los constructores no correspondía con las necesidades de la población de estratos más bajos, y adicionalmente el crecimiento de alrededor del $50 \%$ de la oferta de vivienda se producía fuera de las normas establecidas por el Estado. De la misma manera, Fique (2005) realizó una reflexión sobre el deterioro que presentó la vivienda de interés social a partir de la década de los 90 , encontrando que no es asequible para la población de menores ingresos, sino al estrato promedio; así mismo identificó un incremento relativo de los precios del suelo que causó una desfavorable localización de los proyectos, una sobreexplotación del suelo urbano, y una reducción en la amplitud de las viviendas.

En cuanto a la incapacidad de los hogares para la aprobación de créditos de vivienda, Chiappe (1999) y Escallón (2011) develan esta problemáticas en su estudio. El último autor, en particular, describe los cambios en la política de vivienda en los últimos veinte años, su intencionalidad, condiciones técnicas y financiación, a partir de los cuáles propone cuatro principios generales, retos a la luz de las deficiencias de la política: la vivienda diversa y flexible, en términos de ofrecer múltiples opciones que permitan la asequibilidad a los más pobres; la vivienda suficiente y con calidad, con el fin de disminuir el déficit de vivienda; la vivienda que construye ciudad y la gestión integral, articulada y diversa, en lo referente a atender las dimensiones sociales, financieras, técnicas, culturales y ambientales.

De la misma manera Fique, P. (2008) realizó un análisis del conflicto existente entre dos preceptos del artículo 51 de la Constitución Política de Colombia referentes al «derecho que tienen todos los colombianos a una vivienda digna y la obligación del Estado a hacerlo efectivo», y «la promoción de planes de vivienda y a su adecuada financiación» (pág. 75), identificando una incongruencia en la declaración constitucional y lo planteado en el marco normativo, problemas de los cuales se desprende un permanente deterioro de la calidad y una inadecuada producción de la vivienda.

Por último, se denota la preocupación por la calidad de vida y hábitat de los hogares que acceden a los instrumentos de la política. Camargo, A., \& Hurtado, A. (2011), analizaron la relación entre el concepto de pobreza ${ }^{5}$ y vivienda, y su dinámica para la ciudad de Bogotá, con base en la encuesta de calidad de vida del

4 Este sistema sustituyó al antiguo esquema en el cual el Estado era directamente constructor de viviendas, proveedor de créditos y de subsidios.

5 En este estudio el concepto de pobreza se asocia a la falta de ingresos en los diferentes enfoques multidimensionales. 
año 2007 del DANE. Se identificó la existencia de pobreza inducida por vivienda y nueva pobreza urbana, dado el incremento del gasto en los hogares a partir de la obtención de un predio, planteando una gran necesidad de reorientar estructuralmente las políticas habitacionales, en términos de orientarlas a la reducción de los índices de pobreza.

En este mismo sentido, Agudelo, C. Vaca, M. \& García, C. (2013) analizaron dos de los principales modelos aplicados en Bogotá para atender las necesidades de vivienda de las familias de bajos ingresos, mercado y producción social de hábitat y vivienda (PSHV), observando el avance en la apropiación del concepto de hábitat y no simplemente en términos de construcciones; sin embargo los autores plantean algunos interrogantes para futuras investigaciones, que son aspectos relevantes a contemplar en este proyecto: " $i s e$ debe esperar que el mercado vaya adaptando paulatinamente su respuesta a la demanda de los grupos de bajos ingresos?, ¿será cierto que el mercado es el llamado a resolver definitivamente el problema de vivienda en las ciudades colombianas?, ¿cómo pueden las organizaciones encargadas de manejar procesos de PSHV, mejorar efectivamente las capacidades sociales de las comunidades y entregar no solo vivienda, sino hábitat?» (pág. 50).

Henao, L. Herrera, G. Trujillo, H. \& Cárdenas, A. (2014), describen la evolución histórica de la política de vivienda en Colombia desde 1918 a 2014, informe generado por el Ministerio de Vivienda, Calidad y Territorio para el Sétimo Foro Urbano Mundial; en el cual analiza la ejecución de la política actual, mostrando sus logros e impactos, enfocados principalmente a la provisión de servicios básicos, espacios públicos, equipamiento y sistemas de movilidad.

\subsection{La vivienda como mecanismo para el desarrollo económico y el mejoramiento de la Calidad de Vida}

En el marco de la intervención del Banco Mundial para la recuperación de los países en el periodo posguerra, el economista Lauchlin Currie es enviado a Colombia, en 1949, con el objetivo de diseñar e implementar estrategias macroeconómicas que favorecieran el crecimiento económico y el desarrollo social del país, en especial en las zonas rurales, en donde se evidenciaban las condiciones de vida más precarias (López, 2011, págs. 27-29).

En consecuencia, Currie plantea una estrategia de reactivación económica a través de la denominada «teoría del crecimiento acelerado», que consistió principalmente en incrementar la productividad a través de la reorganización de los recursos existentes en el país, la implementación de nuevas tecnologías, y la focalización de la inversión pública en actividades productivas de impacto social, así como la distribución equitativa del ingreso, un aumento -en términos de cobertura- de la provisión de servicios públicos, el mejoramiento de la estructura de las importaciones en pro de la inversión, y finalmente la estimulación del sector de la construcción, como mecanismo de jalonamiento de la economía y el mejoramiento infraestructural del país (López, 2011, págs. 33-39).

Currie, a partir de sus estudios empíricos del ciclo económico identificó que «existen ciertos sectores que jalonan las fases de expansión y contracción propias del ciclo, arrastrando el desempeño de los demás sectores» (Álvarez 2013, pág. 235). En este sentido, el cumplimento los objetivos anteriormente enunciados se lograrían a través del incremento de la actividad de un sector líder que contara con características como: la permisión para estimularlo exógenamente, la existencia de una demanda potencial, la inflexibilidad para saturar fácilmente el mercado con sus productos, y la vinculación con en un alto nivel con servicios y productos de otros sectores. De esta manera, el sector de la construcción se constituyó en la plataforma perfecta para alcanzar nuevos niveles de desarrollo, a fin de reducir el índice de desempleo de la mano de obra poco calificada e incrementar la demanda de insumos locales. (Montenegro, 2012, pág. 92).

Por otro lado, es de mencionar que Currie concibió el desarrollo como un mecanismo para alcanzar el crecimiento, no en si como una meta, sino como un proceso permanente que conlleva a mayores niveles de bienestar:

"La línea divisoria que yo trazaría entre los países más desarrollados y los menos desarrollados no puede ser establecida en términos de crecimiento del PIB ni tampoco con base en el nivel de ingreso per cápita a su distribución, sino en la creación de lo que parecen ser las condiciones esenciales para ejercer un control significante y consciente sobre el medio ambiente, en bien de la supervivencia en primer lugar, y, en segundo, del bienestar» (Currie, 1966, pág. 185).

No obstante, el tema de la medición de bienestar para Currie (1993), está asociado a la identificación del nivel de satisfacción que los individuos experimentan al alcanzar o poseer algo, teniendo como punto de 
partida el referente construido a partir de la comparación con lo alcanzado por otros seres humanos, dada su naturaleza. De esta manera, los niveles de satisfacción pueden ser diferentes y el nivel de frustración se puede reducir a través del alcance de los objetivos que cada individuo haya propuesto (pág. 185), así como del suplemento de necesidades psíquicas, y actividades no pecuniarias (Currie, pág. 308).

Lo anterior materializa la postura de Currie en dos aspectos relevantes y complementarios: el crecimiento económico y el bienestar, fomentando el desarrollo socioeconómico, y por ende la elevación de las condiciones de vida, en especial para la población más vulnerable. Este enunciado tiene gran relación con los objetivos de las políticas habitacionales actuales. La ley 388 de 1997 fija los lineamientos para el ordenamiento del territorio y encamina uno de sus objetivos a la propensión por el mejoramiento de la calidad de vida de los habitantes, la distribución equitativa de las oportunidades y los beneficios del desarrollo.

Desde esta misma perspectiva, el Plan Nacional de Desarrollo establece como objetivo reducir las brechas poblacionales y territoriales en la provisión de servicios de calidad en salud, educación, servicios públicos, infraestructura y conectividad, así como erradicar la pobreza; lo que en consecuencia conlleva a una visión de desarrollo económico en la cual se evidencie una mejor distribución de la riqueza. La evaluación de dichos aspectos se realiza mediante el modelo aplicado por el Departamento Administrativo Nacional de Estadística DANE, quien para la medición del mejoramiento de la calidad de vida en Colombia, vincula variables relativas a las características infraestructurales de la vivienda (material de paredes, pisos y servicios públicos), educación, salud, cuidado de los niños, fuerza de trabajo, gastos e ingresos, tenencia de bienes, entre otros.

\subsection{Evaluación de la Política}

Las prácticas evaluativas han sido inherentes a la dinámica natural de los programas de vivienda social en Colombia, y han revelado persistentes problemas como el déficit cualitativo y cuantitativo, la falta de asequibilidad de los hogares con menores recursos a las líneas de financiación, la pobreza y la falta de cobertura y focalización de la política; lo que sustenta la necesidad de realizar una aproximación hacia una «evaluación integral», que basándose en los postulados de Currie, logre articular varias dimensiones.

En tanto que Currie consideró que el crecimiento económico del país podría darse mediante la estimulación del sector de la construcción, con una perspectiva social acorde a la época -mejorar las condiciones de vida de la población más vulnerable y así los niveles de bienestar-como mecanismo para fomentar el desarrollo, éste capítulo despliega la propuesta de evaluación, de la siguiente manera:

1. Desarrollo Macroeconómico: se realizó un análisis de diferentes indicadores económicos, a fin de determinar la incidencia del sector de la construcción en el crecimiento económico del país.

2. Probabilidad de tenencia de vivienda a partir del modelo de vulnerabilidad de la Política: teniendo en cuenta que la política de vivienda de interés social se constituye en el mecanismo para favorecer a los hogares en condiciones de vulnerabilidad, con ingresos menores a 4 SMLV; se validó a través de un modelo logit, sí poseer las características contempladas para la asignación de subsidios, ha posibilitado la tenencia de vivienda en este grupo poblacional.

3. Análisis de aspectos de calidad de vida no inherentes a la política: Se revisó el comportamiento de algunos aspectos indagados a través de la encuesta de calidad de vida aplicada por el DANE en los años 2010, 2012 y 2015, con el propósito de identificar problemáticas asociadas a los beneficiarios de subsidios de vivienda durante los últimos 6 años.

4. Percepción del nivel de la calidad de vida: Finalmente, atendiendo a la concepción de Currie sobre el bienestar y su asociación al nivel de satisfacción de los individuos, se realizó un análisis de la apreciación del grupo poblacional de la política, diferenciando a aquellos que han sido beneficiarios, sobre el mejoramiento de sus condiciones de vida, a partir de los resultados de la encuesta aplicada por el DANE en los años 2010, 2012 y 2015.

\subsubsection{Desarrollo Macroeconómico}

En este apartado se presentan algunos datos estadísticos que evidencian los principales resultados de la política habitacional en Colombia y su desarrollo ${ }^{6}$ :

\footnotetext{
6 Datos consultados en https://camacol.co/informacion-economica/construccion-en-cifras.
} 
GAPP. Nueva Época - N. ${ }^{\circ}$ 21, Mayo-Octubre 2019 - ISSN: 1989-8991 - DOI: 10.24965/gapp.v0i21.10522 - [Págs. 125-149]

Evaluación integral de la política pública de vivienda de interés social en Bogotá, 2008-2016

Mayra Alejandra Lozano Rodríguez / Hernán Darío Enríquez Sierra

- Producción de vivienda de interés social con relación al número de subsidios distritales efectivos.

GRÁFICO 1. PRODUCCIÓN DE VIVIENDA DE INTERÉS SOCIAL/ ASIGNACIÓN DE SUBSIDIOS VIP

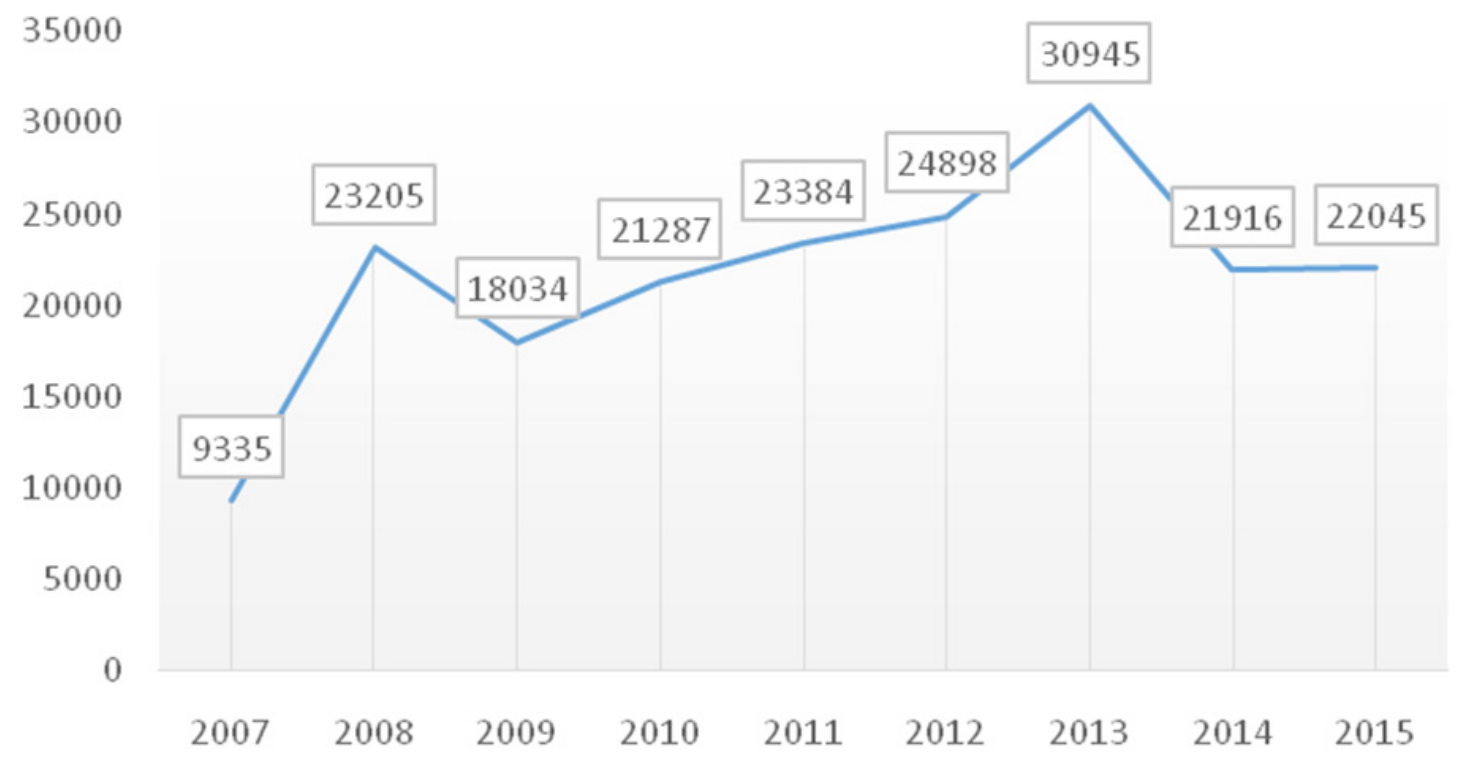

Fuente: CAMACOL, 2016.

En cuanto a la producción de vivienda de interés social, se identifica la culminación en promedio de 21.672 viviendas al año. Si bien no fue posible obtener los datos de los subsidios entregados por los diferentes organismos autorizados en la ciudad Bogotá, en un informe de evaluación de política pública del programa de vivienda y hábitat humanos (2015) de la Secretaría de Hábitat, se reporta que en el período comprendido entre el 2009 y 2012 se asignaron 14.025 subsidios, de los cuáles sólo se desembolsaron 8.971, es decir el $64 \%$ de la aprobación total. La producción de unidades de vivienda para el mismo periodo de tiempo fue de 87.603 (CAMACOL, 2016), lo que indica una asignación de tan sólo el 10,24\% de la producción de vivienda de interés social a la población más vulnerable. Ésta afirmación, teniendo que la Secretaría de Hábitat sólo gestiona los subsidios de los hogares que no cuentan con vinculación a las Cajas de Compensación Familiar, es decir los que no tienen una relación contractual formal.

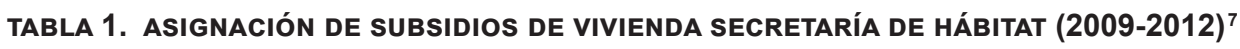

\begin{tabular}{rrrrrrr}
\hline \multicolumn{1}{c}{ 2009-2012 } & $\begin{array}{c}\text { Número } \\
\text { asignado }\end{array}$ & $\begin{array}{c}\text { Número } \\
\text { desembolsado }\end{array}$ & $\begin{array}{c}\text { Porcentaje } \\
\text { desembolsoso }\end{array}$ & $\begin{array}{c}\text { Valor } \\
\text { asignado } \\
\text { (millones) }\end{array}$ & $\begin{array}{c}\text { Valor } \\
\text { desembolsado } \\
\text { (millones) }\end{array}$ & $\begin{array}{c}\text { Porcentaje } \\
\text { desembolsado }\end{array}$ \\
\hline Adquisición de vivienda & 14.025 & 8.971 & $64 \%$ & 158.682 & 101.145 & $63,7 \%$ \\
\hline Arrendamiento & 696 & 465 & $67 \%$ & 1.290 & 1.287 & $99,7 \%$ \\
\hline Mejoramiento de vivienda & 864 & 811 & $94 \%$ & 3.415 & 3.221 & $94,3 \%$ \\
\hline Total & $\mathbf{1 5 . 5 8 5}$ & $\mathbf{1 0 . 2 4 7}$ & $\mathbf{6 6 \%}$ & $\mathbf{1 6 3 . 3 8 8}$ & $\mathbf{1 0 5 . 6 5 2}$ & $\mathbf{6 5 , 0 \%}$ \\
\hline
\end{tabular}

Secretaría de Hábitat, 2015.

- Unidades de vivienda iniciadas por rango de precio. Sectorial.

7 Gráfico tomado de: Secretaría Distrital de Hábitat (2015). Política Distrital del Hábitat. Bogotá: Subdirección de Información 
GRÁFICO 2. UNIDADES DE VIVIENDA INICIADAS POR RANGO DE PRECIO

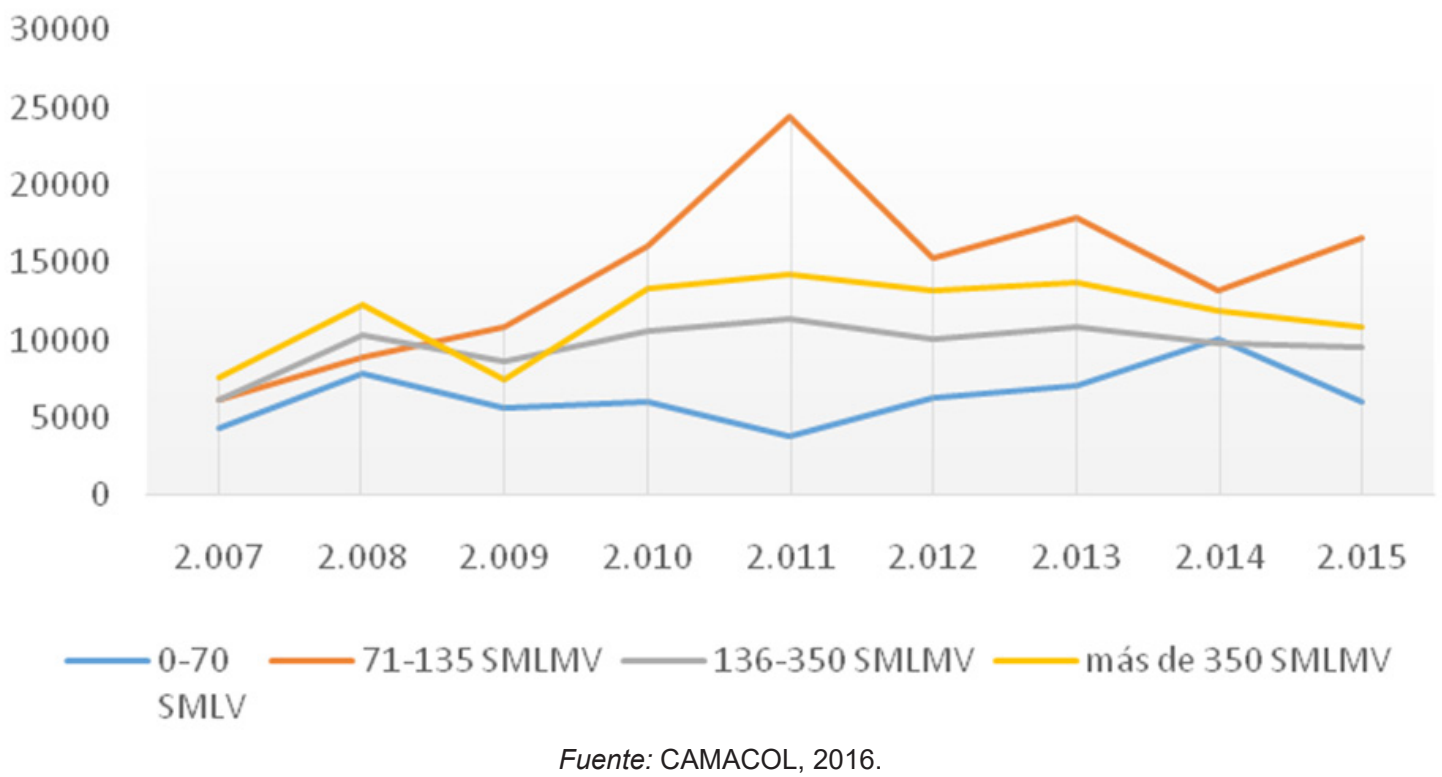

En lo referente a vivienda de interés prioritario se identifica que en promedio se inicia al año la producción de 6.371 unidades. La variación porcentual no es regular, en el 2008 la producción incrementó en un 79\% con relación al año anterior, en el 2009 (-29\%), 2010 (7\%), 2011 (-35\%), 2012 (61\%), 2013 (13\%), $2014(40 \%)$ y $2015(-39 \%)$.

De la misma manera, la vivienda de interés social tiene un promedio de producción anual de 14.404 unidades, presentándose un incremento del 133\%, del 2007 al 2015 En 2008 incrementó un 44\% con relación al año 2007, en el 2009 (22\%), 2010 (47\%), 2011 (51\%), 2012 (-37\%), $2013(17 \%), 2014$ (-26\%), y $2015(26 \%)$

De la producción total de vivienda (de 0-70 a 350 SMLV), las VIS representan el $49 \%$ de la producción, sin embargo tan sólo el $15,11 \%$ corresponde a las VIP.

- Porcentaje de representación del Sector de la Construcción en el PIB de Bogotá.

GRÁFICO 3. \% DEL SECTOR DE LA CONSTRUCCIÓN EN EL PIB

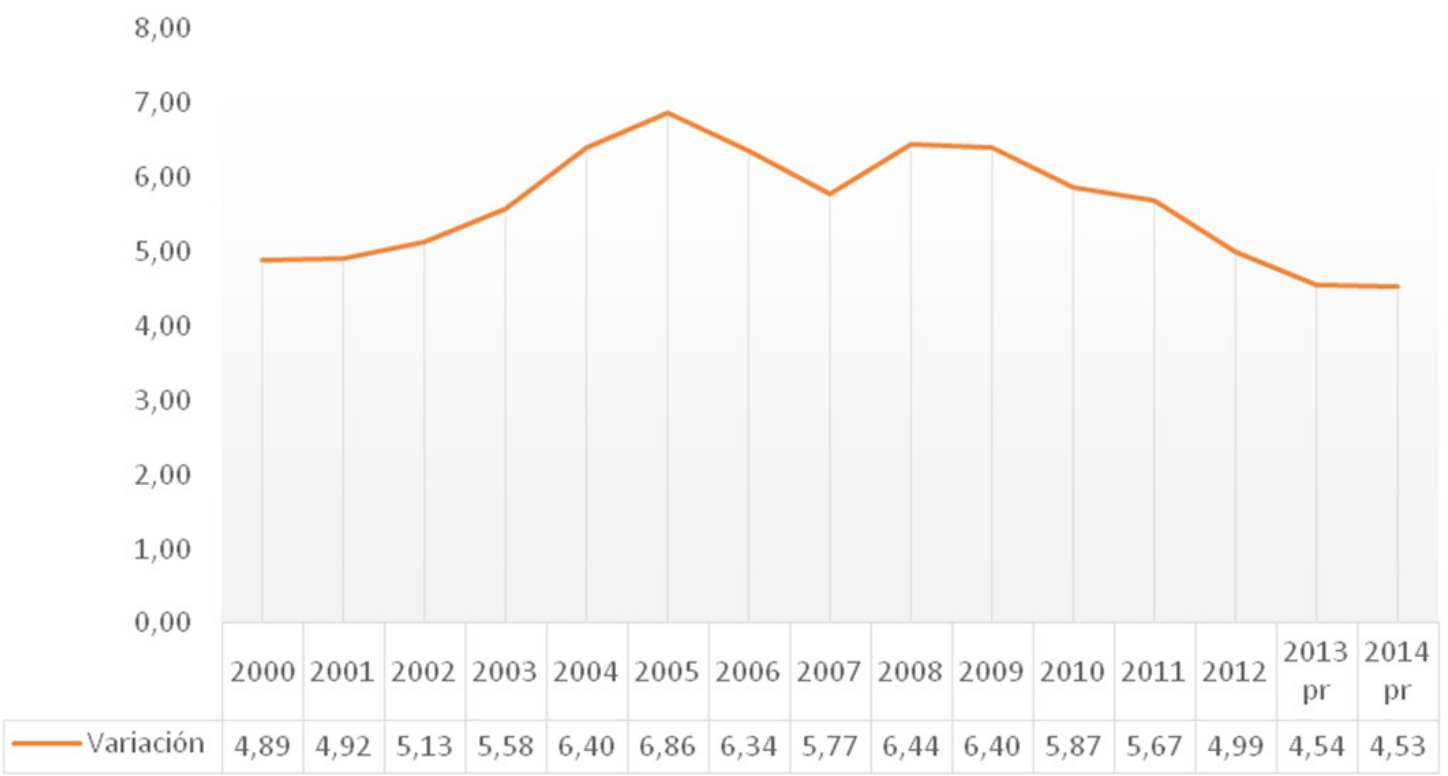

Fuente: CAMACOL, 2016. 
La participación del sector de la construcción en el PIB, el crecimiento ha sido sostenido, con variaciones que van entre el 4,53 y el 6,86. Los mayores incrementos se dan entre el $2004(6,40)$ y el $2009(6,40)$, los cuales pueden relacionarse directamente con la implementación del microcrédito inmobiliario y la creación del Fondo Nacional de Vivienda, en el periodo presidencial de Álvaro Uribe.

- Ocupación en el sector de la construcción en Bogotá.

\section{GRÁFICO 4. \% DE OCUPACIÓN EN EL SECTOR DE LA CONSTRUCCIÓN CON RELACIÓN AL NÚMERO TOTAL DE OCUPADOS}

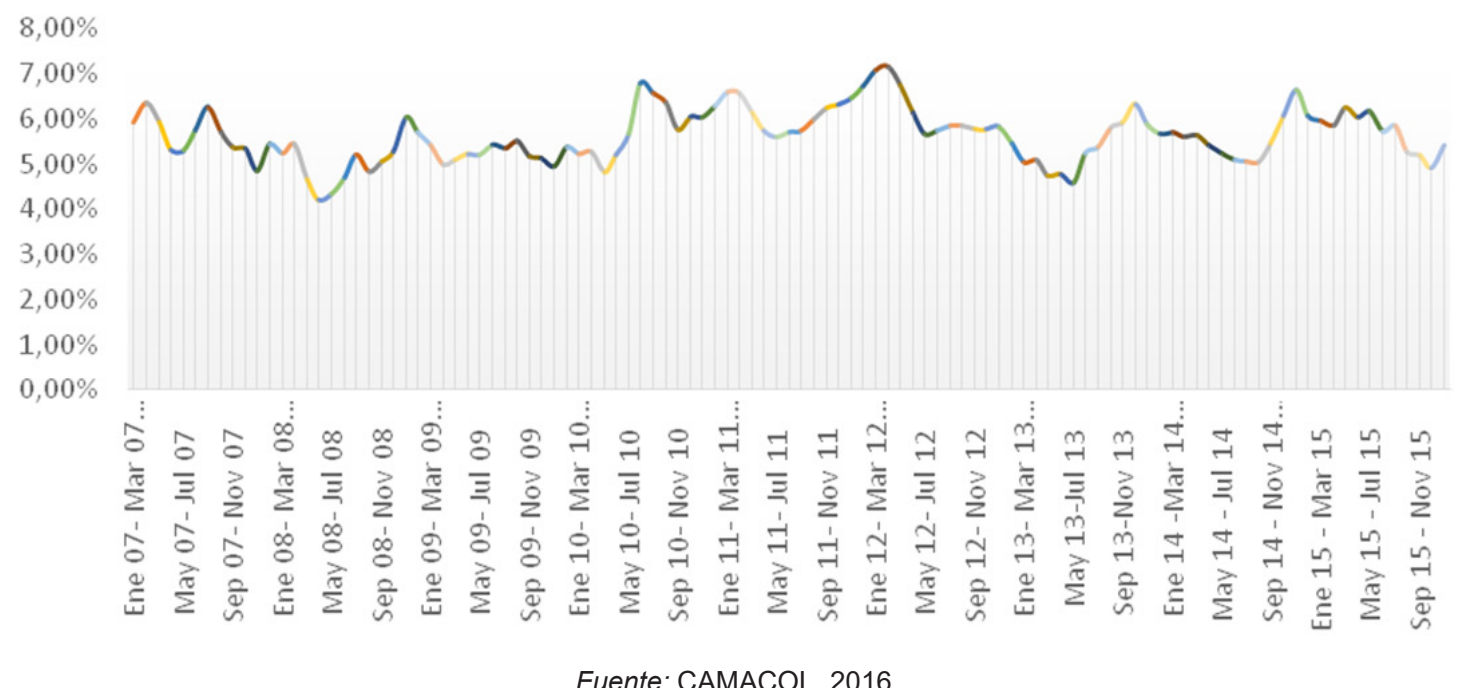

A pesar del incremento en la construcción de unidades de vivienda, no se evidencian cambios significativos con relación a la generación de empleo. En general la participación de la ocupación del sector de la construcción respecto al número de ocupados total en la ciudad de Bogotá, oscila entre un $5 \%$ y $7 \%$.

- Variación anual del índice de costos de la construcción, con relación a la variación del índice de precios de precios de vivienda nueva en la ciudad de Bogotá.

\section{GRÁFICO 5. VARIACIÓN ANUAL DEL ICC/ VARIACIÓN DEL IPV}

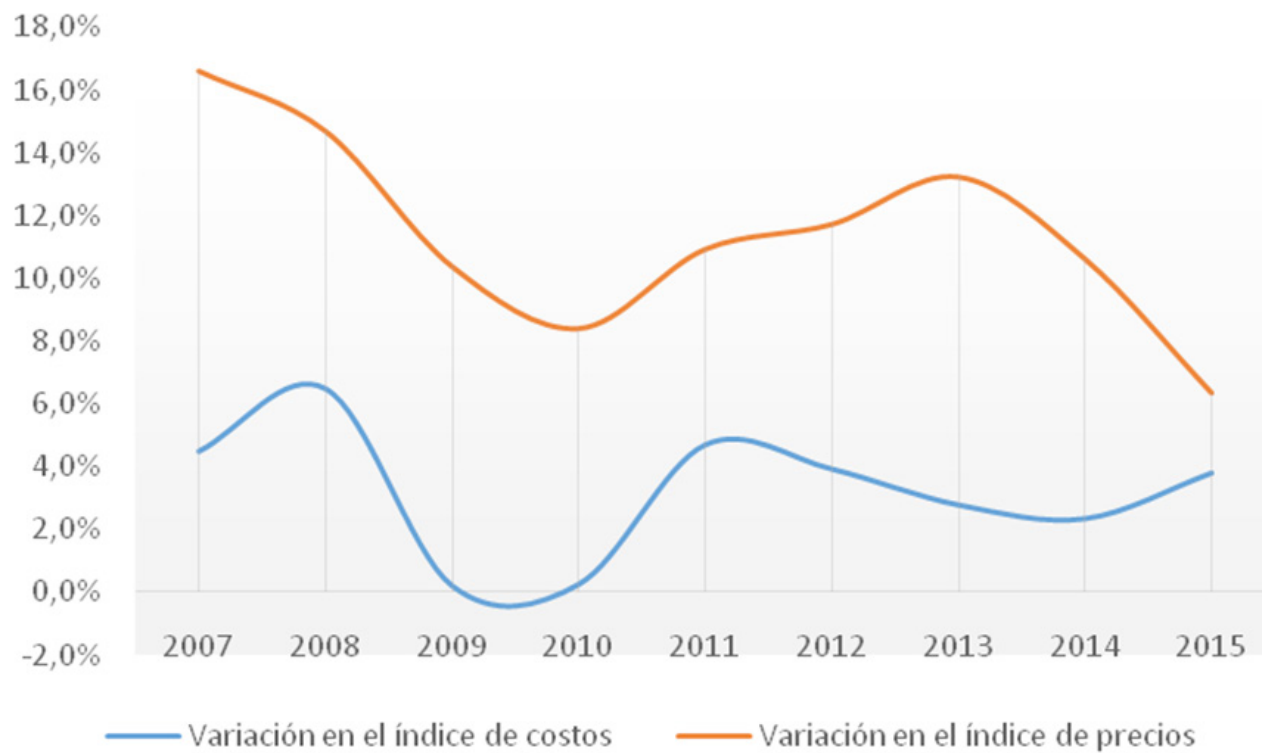

Fuente: CAMACOL, 2016. 
Durante el periodo comprendido entre los años 2008 y 2011, se refleja un comportamiento casi proporcional entre la variación del índice de costos y el índice de precios de vivienda nueva en Bogotá; sin embargo a partir del año 2012 se evidencia un crecimiento en mayor proporción del índice de precios con relación al índice de costos. Esto muestra una problemática en la elevación de precios que incide directamente en la oferta de vivienda de interés social y en la capacidad de la población más vulnerable para asumir los requisitos de esta nueva oferta.

Al respecto la Secretaria de Hábitat (2015) explica:

«Este incremento de precios es un fenómeno nacional, pero se ha presentado de forma más pronunciada en la ciudad de Bogotá. De hecho, en el periodo diciembre 2014 - abril de 2015, en la ciudad de Bogotá los precios de la vivienda nueva se han incrementado en promedio un $40 \%$ más en comparación con el agregado del país.» (pág. 22).

Sin duda la elevación de los precios incide directamente en el déficit cuantitativo.

\subsubsection{Probabilidad de tenencia de vivienda a partir del modelo de vulnerabilidad de la Política}

El propósito de este apartado consiste en identificar la probabilidad de tenencia de vivienda propia en los hogares con ingresos menores a 4 salarios mínimos, a partir de las características tenidas en cuenta para la asignación de subsidios distritales, según el modelo de vulnerabilidad de la política de vivienda de interés social, es decir las disposiciones de la resolución 844 de 2014:

GRÁFICO 6. MODELO DE VULNERABILIDAD SDHB

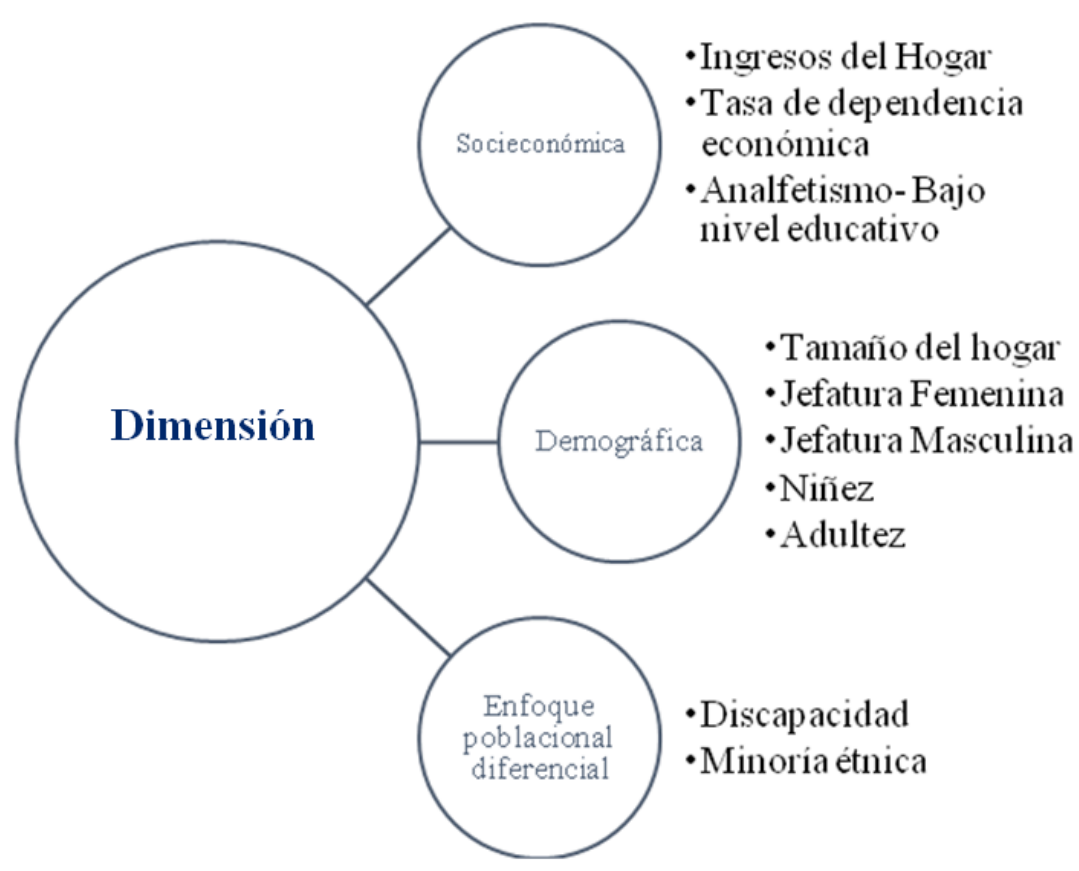

Fuente: Elaboración propia, 2016

En coherencia con lo anterior, se extrajeron de la encuesta de calidad de vida (años) la información referente a las variables: ingreso del hogar (en pesos colombianos), cantidad de personas que componen el hogar (número de personas), subsidio ${ }^{8}$ (var. dummy: 1 sí el hogar fue beneficiario, 0 en caso que no), presencia del padre en el hogar (var. dummy: 1 si el padre convive en el hogar, 0 en caso que no), presencia de la madre (var. dummy: 1 si la madre convive en el hogar, 0 en caso que no), etnia (var. dummy: 1 si existe

\footnotetext{
8 Es de mencionar que los subsidios se reciben en dinero o en especie (materiales para la construcción o mano de obra, para lo cual se debe hacer una estimación del valor del bien o servicio si hubiese tenido que pagarlo, es decir, a precios de mercado).
} 
GAPP. Nueva Época - N. ${ }^{\circ}$ 21, Mayo-Octubre 2019 - ISSN: 1989-8991 - DOI: 10.24965/gapp.v0i21.10522 - [Págs. 125-149]

Evaluación integral de la política pública de vivienda de interés social en Bogotá, 2008-2016 Mayra Alejandra Lozano Rodríguez / Hernán Darío Enríquez Sierra

algún miembro del hogar perteneciente a algún grupo étnico, 0 en caso que no), nivel educativo máximo alcanzado por la madre (var. dummy: 1 según corresponda, primaria completa o incompleta, secundaria completa o incompleta, y superior completa o incompleta, y 0 en caso de no contar con ninguno de los niveles de formación establecidos), nivel educativo máximo alcanzado por el padre (var. dummy: 1 según corresponda, primaria completa o incompleta, secundaria completa o incompleta, y superior completa o incompleta, y 0 en caso de no contar con ninguno de los niveles de formación establecidos). Así mismo, se realizó la comparación de tres años $(2010,2012$ y 2015), con el fin de identificar la incidencia del modelo de vulnerabilidad en la tenencia de vivienda y efectos de mejora en la población objeto, así:

TABLA 2. ESTADÍSTICAS DESCRIPTIVAS VARIABLES CORRESPONDIENTES AL AÑo 2010

\begin{tabular}{|c|c|c|c|c|c|}
\hline Variable & Obs* & Mean & Std. Dev & Min & Max \\
\hline Vivienda Propia & 730 & ,3616438 & ,4808058 & 0 & 1 \\
\hline Ingreso del hogar & 730 & 10,41678 & 5,232284 & 0 & 20,55 \\
\hline Cantidad de personas en el hogar & 730 & 3,257534 & 1,664436 & 1 & 12 \\
\hline Subsidio & 730 & ,0054795 & ,0738709 & 0 & 1 \\
\hline Presencia del padre en el hogar & 730 & ,4471879 & ,4975444 & 0 & 1 \\
\hline Presencia de la madre en el hogar & 730 & ,7037037 & ,4569368 & 0 & 1 \\
\hline Etnia & 730 & ,037037 & ,1889822 & 0 & 1 \\
\hline Nivel educativo máximo alcanzado por la madre (primaria) & 730 & ,6652949 & ,4722112 & 0 & 1 \\
\hline Nivel educativo máximo alcanzado por la madre (secundaria) & 730 & ,2194787 & ,4141777 & 0 & 1 \\
\hline Nivel educativo máximo alcanzado por la madre (universitaria) & 730 & ,0301783 & ,1711952 & 0 & 1 \\
\hline Nivel educativo máximo alcanzado por el padre (primaria) & 730 & 6748971 & ,4687349 & 0 & 1 \\
\hline Nivel educativo máximo alcanzado por el padre (secundaria) & 730 & ,3017833 & ,4593469 & 0 & 1 \\
\hline Nivel educativo máximo alcanzado por el padre (universitaria) & 730 & 0548697 & 2278821 & 0 & 1 \\
\hline
\end{tabular}

* Aplicando el factor de expansión a la muestra, las apreciaciones realizadas corresponden a 1.304.546 hogares con ingresos menores a 4 SMLV.

Fuente: Elaboración propia, 2016.

TABLA 3. ESTADÍSTICAS DESCRIPTIVAS VARIABLES CORRESPONDIENTES AL AÑo 2012

\begin{tabular}{|c|c|c|c|c|c|}
\hline Variable & Obs* & Mean & Std. Dev & Min & Max \\
\hline Vivienda propia & 1136 & ,3723592 & ,4836463 & 0 & 1 \\
\hline Ingreso del hogar & 1136 & 11,17026 & 5,942882 & 0 & 22,63833 \\
\hline Cantidad de personas en el hogar & 1136 & 3,053697 & 1,519832 & 1 & 12 \\
\hline Subsidio & 1136 & ,0088028 & ,0934506 & 0 & 1 \\
\hline Presencia del padre en el hogar & 1136 & , 1035242 & ,4908204 & 0 & 1 \\
\hline Presencia de la madre en el hogar & 1136 & ,6784141 & ,4672909 & 0 & 1 \\
\hline Etnia & 1136 & ,0819383 & ,2743916 & 0 & 1 \\
\hline $\begin{array}{l}\text { Nivel educativo máximo alcanzado por la madre } \\
\text { (primaria) }\end{array}$ & 1136 & ,6220264 & ,4850947 & 0 & 1 \\
\hline $\begin{array}{l}\text { Nivel educativo máximo alcanzado por la madre } \\
\text { (secundaria) }\end{array}$ & 1136 & ,245815 & ,4307592 & 0 & 1 \\
\hline
\end{tabular}


GAPP. Nueva Época - N.21, Mayo-Octubre 2019 - ISSN: 1989-8991 - DOI: 10.24965/gapp.v0i21.10522 - [Págs. 125-149]

Evaluación integral de la política pública de vivienda de interés social en Bogotá, 2008-2016 Mayra Alejandra Lozano Rodríguez / Hernán Darío Enríquez Sierra

\begin{tabular}{|c|c|c|c|c|c|}
\hline Variable & Obs* & Mean & Std. Dev & Min & Max \\
\hline $\begin{array}{l}\text { Nivel educativo máximo alcanzado por la madre } \\
\text { (universitaria) }\end{array}$ & 1136 & ,0669604 & ,2500635 & 0 & 1 \\
\hline $\begin{array}{l}\text { Nivel educativo máximo alcanzado por el padre } \\
\text { (primaria) }\end{array}$ & 1136 & ,6164021 & ,4864764 & 0 & 1 \\
\hline $\begin{array}{l}\text { Nivel educativo máximo alcanzado por el padre } \\
\text { (secundaria) }\end{array}$ & 1136 & ,3183422 & ,4660386 & 0 & 1 \\
\hline $\begin{array}{l}\text { Nivel educativo máximo alcanzado por el padre } \\
\text { (universitaria) }\end{array}$ & 1136 & ,1393298 & ,3464431 & 0 & 1 \\
\hline
\end{tabular}

Fuente: Elaboración propia, 2016.

TABLA 4. ESTADÍSTICAS DESCRIPTIVAS VARIABLES CORRESPONDIENTES AL AÑO 2015

\begin{tabular}{|c|c|c|c|c|c|}
\hline Variable & Obs* & Mean & Std. Dev & Min & Max \\
\hline Vivienda propia & 1235 & ,394332 & ,4889047 & 0 & 1 \\
\hline Ingreso del hogar & 1235 & 12,21835 & 6,720833 & 0 & 25,75587 \\
\hline Cantidad de personas en el hogar & 1235 & 3,105263 & 1,541515 & 1 & 12 \\
\hline Subsidio & 1235 & ,0097166 & ,0981325 & 0 & 1 \\
\hline Presencia del padre en el hogar & 1235 & ,4234818 & ,4943104 & 0 & 1 \\
\hline Presencia de la madre en el hogar & 1235 & ,697166 & ,4596702 & 0 & 1 \\
\hline Etnia & 1235 & 0,834008 & ,2765991 & 0 & 1 \\
\hline $\begin{array}{l}\text { Nivel educativo máximo alcanzado por la madre } \\
\text { (primaria) }\end{array}$ & 1235 & 6437247 & ,4790919 & 0 & 1 \\
\hline $\begin{array}{l}\text { Nivel educativo máximo alcanzado por la madre } \\
\text { (secundaria) }\end{array}$ & 1235 & ,2582996 & ,4378769 & 0 & 1 \\
\hline $\begin{array}{l}\text { Nivel educativo máximo alcanzado por la madre } \\
\text { (universitaria) }\end{array}$ & 1235 & 0725745 & ,2600357 & 0 & 1 \\
\hline $\begin{array}{l}\text { Nivel educativo máximo alcanzado por el padre } \\
\text { (primaria) }\end{array}$ & 1235 & ,6337115 & ,4819849 & 0 & 1 \\
\hline $\begin{array}{l}\text { Nivel educativo máximo alcanzado por el padre } \\
\text { (secundaria) }\end{array}$ & 1235 & ,3306321 & ,4706315 & 0 & 1 \\
\hline $\begin{array}{l}\text { Nivel educativo máximo alcanzado por el padre } \\
\text { (universitaria) }\end{array}$ & 1235 & , 149919 & ,3571367 & 0 & 1 \\
\hline
\end{tabular}

Fuente: Elaboración propia, 2016.

Para hallar la probabilidad de tenencia de vivienda en los hogares con ingresos menores a 4 SMLV, dadas algunas de las características del modelo de vulnerabilidad para la asignación de subsidios, se utilizó un modelo logit ${ }^{9}$, el cual presenta la siguiente forma, partiendo de una función de distribución logística:

$$
\text { 1. } f(z)=\frac{1}{1+e^{-z}}=\frac{1}{1+\frac{1}{e^{z}}}=\frac{e^{z}}{1+e^{z}}
$$

Acotada entre 0 y 1 , teniendo en cuenta:

$$
\lim _{z \rightarrow-\infty} f(z)=0 \quad \lim _{z \rightarrow-\infty} f(z)=1
$$

${ }_{9}$ Consultado en: http://www.ugr.es/ romansg/material/WebEco/Eco2-Discreta.pdf. 
GAPP. Nueva Época - N. 21, Mayo-Octubre 2019 - ISSN: 1989-8991 - DOI: 10.24965/gapp.v0i21.10522 - [Págs. 125-149]

Evaluación integral de la política pública de vivienda de interés social en Bogotá, 2008-2016 Mayra Alejandra Lozano Rodríguez / Hernán Darío Enríquez Sierra

La forma del modelo es:

$$
\text { 2. } Y i=f(Z i)+u i, i=1, \cdots, n
$$

Donde, $f(z)$ es la probabilidad de tenencia de vivienda y $Z i=\beta 1+\beta 2 X 2 i+\ldots+\beta k X k i$, dados los valores de las variables independientes $X 2, \ldots . . X k$.

En la cual Zi corresponde a la tenencia de vivienda propia, y $X 2 \ldots X \mathrm{~K}$, corresponde a las variables: ingreso del hogar, cantidad de personas en el hogar, subsidio, presencia del padre en el hogar, presencia de la madre del hogar, pertenencia a un grupo étnico, nivel educativo del padre y nivel educativo de la madre.

La interpretación se realiza a través de los cambios marginales, teniendo en cuenta que el modelo no es lineal:

$$
\text { 3. } \frac{\partial Y i}{\partial X j i}=\frac{e^{-Z i}}{\left(1+e^{-Z i}\right)^{2}} B j, j=1, \ldots, k
$$

Es de mencionar, que además de aplicar el modelo en los tres años seleccionados, se establecieron diferentes perfiles de hogares, intentando combinar las múltiples variables y evidenciar su incidencia en la posesión de vivienda propia.

Perfil 1: Hogares con ingresos no superiores a 4 salarios mínimos legales y nivel máximo educativo alcanzado por los padres «primaria».

TABLA 5. MODELO DE PROBABILIDAD DE TENENCIA DE VIVIENDA,

\begin{tabular}{|c|c|c|c|}
\hline Variable & $\begin{array}{l}\text { Cambios marginales } \\
2010\end{array}$ & $\begin{array}{l}\text { Cambios marginales } \\
2012\end{array}$ & $\begin{array}{c}\text { Cambios marginales } \\
2015\end{array}$ \\
\hline Ingreso del hogar (hasta 4 smlv) &, $0220285^{\star *}$ & ,0078299 &, $0101112^{* *}$ \\
\hline Cantidad de personas en el hogar & ,0142438 &,- 005545 &,- 0104556 \\
\hline Subsidio & ,1502438 &, $3735838^{* * *}$ & $274832^{* * *}$ \\
\hline Presencia del padre en el hogar &, 0460248 &,- 0018191 &,- 0031694 \\
\hline Presencia de la madre en el hogar &,- 0950188 &,- 0132407 &,- 00416 \\
\hline Etnia &,- 0405796 &,- 0026165 &,- 0153068 \\
\hline $\begin{array}{l}\text { Nivel educativo máximo alcanzado } \\
\text { por la madre (primaria) }\end{array}$ &,- 0588153 &,- 0156407 &,- 0148302 \\
\hline \multirow[t]{2}{*}{$\begin{array}{l}\text { Nivel educativo máximo alcanzado } \\
\text { por el padre (primaria) }\end{array}$} &,- 0168769 &,- 020568 &,- 0164811 \\
\hline & $\begin{array}{l}\text { Núm. obs. } 729 \\
\text { Chi 2: } 52,38 \\
\text { Prob>chi2: } 0,0000 \\
\text { Pseudo R2: } 0,0549 \\
\text { Estat classification- } \\
\text { correctly classified: } \\
64,61 \%\end{array}$ & $\begin{array}{l}\text { Núm. obs. } 1134 \\
\text { Chi 2: } 57,66 \\
\text { Prob>chi2: } 0,0000 \\
\text { Pseudo R2: } 0,0385 \\
\text { Estat classification- } \\
\text { correctly classified: } \\
63,76 \%\end{array}$ & $\begin{array}{l}\text { Núm. obs. } 1234 \\
\text { Chi 2: } 73,42 \\
\text { Prob>chi2: } 0,0000 \\
\text { Pseudo R2: } 0,0444 \\
\text { Estat classification- } \\
\text { correctly classified: } \\
63,45 \%\end{array}$ \\
\hline
\end{tabular}
DADAS LAS CARACTERÍSTICAS DE LOS HOGARES CON EL PERFIL 1

* Significativas al $10 \%$. ${ }^{* *}$ Significancia al $5 \%$. ${ }^{* * *}$ Significancia al $1 \%$.

Fuente: Construcción Propia.

El primer perfil corresponde a un hogar con ingresos de hasta 4 SMLV y un número de integrantes promedio (4 personas), en el que la primaria, completa o incompleta es el nivel máximo educativo alcanzado por sus padres. En este caso se identificó que el principal determinante para la tenencia de vivienda, dadas las características enunciadas, es el ingreso. De esta manera un incremento en el ingreso en 100.000 \$, en promedio y ceteris paribus, aumentó la probabilidad de tenencia de vivienda en un $2,22 \%$ en el 2010 y en un $1,01 \%$ en el 2015.

Igualmente los hogares que accedieron al subsidio aumentaron la probabilidad de tenencia de vivienda en un $37,35 \%$ en el año 2012 y $27,48 \%$ en el año 2015 , frente a quienes no fueron beneficiarios. 
GAPP. Nueva Época - N. 21, Mayo-Octubre 2019 - ISSN: 1989-8991 - DOI: 10.24965/gapp.v0i21.10522 - [Págs. 125-149]

Evaluación integral de la política pública de vivienda de interés social en Bogotá, 2008-2016 Mayra Alejandra Lozano Rodríguez / Hernán Darío Enríquez Sierra

Perfil 2: Hogares con ingresos no superiores a 2 salarios mínimos legales y nivel máximo educativo alcanzado por los padres "primaria».

TABLA 6. MODELO DE PROBABILIDAD DE TENENCIA DE VIVIENDA, DADAS LAS CARACTERÍSTICAS DE LOS HOGARES CON EL PERFIL 2

\begin{tabular}{|c|c|c|c|}
\hline Variable & $\begin{array}{l}\text { Cambios marginales } \\
2010\end{array}$ & $\begin{array}{l}\text { Cambios marginales } \\
2012\end{array}$ & $\begin{array}{c}\text { Cambios marginales } \\
2015\end{array}$ \\
\hline Ingreso del hogar (hasta 2 smlv) &, $0249592^{* * *}$ &, $0139751^{* *}$ &, $171898^{* * *}$ \\
\hline Cantidad de personas en el hogar & 01611388 &,- 0098969 &,- 0177918 \\
\hline Subsidio &, 1448164 & ,4592998*** &, $3401398^{* *}$ \\
\hline Presencia del padre en el hogar & 0494879 &,- 0032539 &,- 0054115 \\
\hline Presencia de la madre en el hogar &,$- 1210994^{* *}$ &,- 0240169 &,- 0071253 \\
\hline Etnia &,- 048266 &,- 0046849 &,- 0265758 \\
\hline $\begin{array}{l}\text { Nivel educativo máximo alcanzado } \\
\text { por la madre (primaria) }\end{array}$ &,- 0715556 &,- 0284541 &,- 0257313 \\
\hline \multirow[t]{2}{*}{$\begin{array}{l}\text { Nivel educativo máximo alcanzado } \\
\text { por el padre (primaria) }\end{array}$} &,- 0195068 &,- 0376467 &,- 0286613 \\
\hline & $\begin{array}{l}\text { Núm. obs. } 729 \\
\text { Chi 2: } 52,38 \\
\text { Prob>chi2: } 0,0000 \\
\text { Pseudo R2: } 0,0549 \\
\text { Estat classification- } \\
\text { correctly classified: } \\
64,61 \%\end{array}$ & $\begin{array}{l}\text { Núm. obs. } 1134 \\
\text { Chi 2: } 57,66 \\
\text { Prob>chi2: } 0,0000 \\
\text { Pseudo R2: } 0,0385 \\
\text { Estat classification- } \\
\text { correctly classified: } \\
63,76 \%\end{array}$ & $\begin{array}{l}\text { Núm. obs. } 1234 \\
\text { Chi 2: } 73,42 \\
\text { Prob>chi2: } 0,0000 \\
\text { Pseudo R2: } 0,0444 \\
\text { Estat classification- } \\
\text { correctly classified: } \\
63,45 \%\end{array}$ \\
\hline
\end{tabular}

${ }^{*}$ Significativas al $10 \% .{ }^{* *}$ Significancia al $5 \% .{ }^{* * *}$ Significancia al $1 \%$.

Fuente: Construcción Propia.

El segundo perfil corresponde a un hogar con ingresos de hasta 2 SMLV, constituido por 4 personas, en el que la primaria (completa o incompleta) es el nivel máximo de escolaridad alcanzado por los padres. Se identificó nuevamente que los principales determinantes para la tenencia de vivienda en este tipo de hogares es el ingreso y el subsidio. En este sentido, ante un incremento de $100.000 \$$ en el ingreso, la probabilidad de poseer vivienda propia aumentó en un 2,49\% en el 2010, 1,39\% en el 2011 y en un 17,18\% en el 2015.

De igual manera, los hogares que fueron beneficiados con el subsidio de vivienda aumentaron la probabilidad de tenencia, del $45 \%$ en el año 2012 y el $34 \%$ en el 2015 , frente a quienes no recibieron subsidio.

Perfil 3: Hogares con ingresos no superiores a 4 salarios mínimos legales y nivel máximo educativo alcanzado por los padres «secundaria».

TABLA 7. MODELO DE PROBABILIDAD DE TENENCIA DE VIVIENDA,

DADAS LAS CARACTERÍSTICAS DE LOS HOGARES CON EL PERFIL 3

\begin{tabular}{lccc}
\hline \multicolumn{1}{c}{ Variable } & Cambios marginales & Cambios marginales & \multicolumn{2}{c}{ Cambios marginales } \\
2015
\end{tabular}


GAPP. Nueva Época - N. 21, Mayo-Octubre 2019 - ISSN: 1989-8991 - DOI: 10.24965/gapp.v0i21.10522 - [Págs. 125-149]

Evaluación integral de la política pública de vivienda de interés social en Bogotá, 2008-2016 Mayra Alejandra Lozano Rodríguez / Hernán Darío Enríquez Sierra

\begin{tabular}{|c|c|c|c|}
\hline Variable & $\begin{array}{l}\text { Cambios marginales } \\
2010\end{array}$ & $\begin{array}{l}\text { Cambios marginales } \\
2012\end{array}$ & $\begin{array}{c}\text { Cambios marginales } \\
2015\end{array}$ \\
\hline Presencia del padre en el hogar & ,0305109 &,- 0109619 &,- 0155156 \\
\hline Presencia de la madre en el hogar &,$- 1295653^{* *}$ &,- 0265443 &,- 0149303 \\
\hline Etnia &,- 0605357 &,- 0055644 &,- 0221934 \\
\hline $\begin{array}{l}\text { Nivel educativo máximo alcanzado } \\
\text { por la madre (secundaria) }\end{array}$ &,$- 1680855^{\star * *}$ &,- 0613728 &,$- 0845147^{*}$ \\
\hline \multirow[t]{2}{*}{$\begin{array}{l}\text { Nivel educativo máximo alcanzado } \\
\text { por el padre (secundaria) }\end{array}$} &,- 0558478 &,- 0243627 &,- 0385401 \\
\hline & $\begin{array}{l}\text { Núm. obs. } 729 \\
\text { Chi 2: } 64,74 \\
\text { Prob>chi2: } 0,0000 \\
\text { Pseudo R2: } 0,0678 \\
\text { Estat classification- } \\
\text { correctly classified: } \\
65,43 \%\end{array}$ & $\begin{array}{l}\text { Núm. obs. } 1134 \\
\text { Chi 2: } 66,33 \\
\text { Prob>chi2: } 0,0000 \\
\text { Pseudo R2: } 0,0443 \\
\text { Estat classification- } \\
\text { correctly classified: } \\
64,81 \%\end{array}$ & $\begin{array}{l}\text { Núm. obs. } 1234 \\
\text { Chi 2: } 90,65 \\
\text { Prob>chi2: } 0,0000 \\
\text { Pseudo R2: } 0,0546 \\
\text { Estat classification- } \\
\text { correctly classified: } \\
64,51 \%\end{array}$ \\
\hline
\end{tabular}

* Significativas al $10 \% .{ }^{* *}$ Significancia al $5 \% .{ }^{* * *}$ Significancia al $1 \%$.

Fuente: Construcción Propia.

El tercer perfil corresponde a un hogar con ingresos de hasta 4 SMLV, con un promedio de integrantes de 4 personas, en el que el nivel educativo máximo alcanzado por sus padres es «secundaria», completa o incompleta. En este caso el ingreso y el subsidio son los principales determinantes de la tenencia de vivienda. Ante un incremento de 100.000 \$ en el ingreso, la probabilidad aumentó en $2,63 \%$ en el $2010,1,14 \%$ y 1,49 en el 2012 y 2015).

Los hogares beneficiaros de subsidio de vivienda aumentaron la probabilidad de tenencia en un $40,84 \%$ en el año 2012 y $29.73 \%$ en el año 2015 , frente a los que no accedieron a él. Igualmente, el hecho de que la madre sólo posea estudios secundarios, hace que la probabilidad de tenencia en el hogar descienda en un $16,81 \%$ en el 2010 y en un $8,451 \%$ en el 2015 , frente e a los hogares en los que la madre posee niveles educativos más altos.

Perfil 4: Hogares con ingresos no superiores a 2 salarios mínimos legales y nivel máximo educativo alcanzado por los padres «secundaria».

TABLA 8. MODELO DE PROBABILIDAD DE TENENCIA DE VIVIENDA, DADAS LAS CARACTERÍSTICAS DE LOS HOGARES CON EL PERFIL 4

\begin{tabular}{lccc}
\hline \multicolumn{1}{c}{ Variable } & Cambios marginales & Cambios marginales & Cambios marginales \\
& $\mathbf{2 0 1 0}$ & $\mathbf{2 0 1 2}$ &, $0201276^{* * *}$ \\
\hline Ingreso del hogar (2 smlv) &, $0202826^{*}$ &, $0178251^{* * *}$ &,- 0167345 \\
\hline Cantidad de personas en el hogar &, 0152809 &,- 0102358 &, $2916731^{* *}$ \\
\hline Subsidio &, 0931231 &, 4309065 &,- 021355 \\
\hline Presencia del padre en el hogar &, 0227631 &,- 0172235 &,- 0205339 \\
\hline Presencia de la madre en el hogar &,- 0114462 &,- 0424791 &,- 030811 \\
\hline Etnia &,- 0495453 &,- 0086882 &,- 1276696 \\
\hline $\begin{array}{l}\text { Nivel educativo máximo alcanzado } \\
\text { por la madre (secundaria) }\end{array}$ &,,$- 155376 * *$ &,$- 1024555^{\star *}$ & \\
\hline
\end{tabular}


GAPP. Nueva Época - N. 21, Mayo-Octubre 2019 - ISSN: 1989-8991 - DOI: 10.24965/gapp.v0i21.10522 - [Págs. 125-149]

Evaluación integral de la política pública de vivienda de interés social en Bogotá, 2008-2016 Mayra Alejandra Lozano Rodríguez / Hernán Darío Enríquez Sierra

\begin{tabular}{lccc}
\hline \multicolumn{1}{c}{ Variable } & $\begin{array}{c}\text { Cambios marginales } \\
\mathbf{2 0 1 0}\end{array}$ & $\begin{array}{c}\text { Cambios marginales } \\
\mathbf{2 0 1 2}\end{array}$ & $\begin{array}{c}\text { Cambios marginales } \\
\mathbf{2 0 1 5}\end{array}$ \\
\hline $\begin{array}{l}\text { Nivel educativo máximo alcanzado } \\
\text { por el padre (secundaria) }\end{array}$ &,- 0454813 &,- 0388871 &,- 0546663 \\
& Núm. obs. 729 & Núm. obs. 1134 & Núm. obs. 1234 \\
& Chi 2: 64,74 & Chi 2: 66,33 & Chi 2: 90,65 \\
& Prob>chi2: 0,0000 & Prob>chi2: 0,0000 & Prob>chi2: 0,0000 \\
& Pseudo R2: 0,0678 & Pseudo R2: 0,0443 & Pseudo R2: 0,0546 \\
& Estat classification- & Estat classification- & Estat classification- \\
& correctly classified: & correctly classified: & correctly classified: \\
& $65,43 \%$ & $64,81 \%$ & \\
\hline
\end{tabular}

Fuente: Construcción Propia.

El siguiente perfil corresponde a un hogar con ingresos de hasta 2 SMLV, conformado por 4 integrantes, en el cual el máximo nivel educativo alcanzado por los padres es «secundaria», completa o incompleta. Nuevamente el ingreso y el subsidio son los principales determinantes de la tenencia de vivienda. Ante un incremento de 100.000 \$ en el ingreso, la probabilidad aumentó en 2,03\% en el 2010, 2,01\% en el 2015; y un $1,78 \%$ en el 2012.

Cuando la madre sólo posee estudios secundarios, la probabilidad de tenencia en el hogar descendió un $15,54 \%$ en el 2010 y un $10,24 \%$ en el 2015 , frente e a los hogares en los que la madre posee niveles educativos más altos.

Perfil 5: Hogares con ingresos no superiores a 4 salarios mínimos legales y nivel máximo educativo alcanzado por los padres «superior».

TABLA 9. MODELO DE PROBABILIDAD DE TENENCIA DE VIVIENDA, DADAS LAS CARACTERÍSTICAS DE LOS HOGARES CON EL PERFIL 5

\begin{tabular}{|c|c|c|c|}
\hline Variable & $\begin{array}{l}\text { Cambios marginales } \\
2010\end{array}$ & $\begin{array}{l}\text { Cambios marginales } \\
\qquad 2012\end{array}$ & $\begin{array}{c}\text { Cambios marginales } \\
2015\end{array}$ \\
\hline Ingreso del hogar (4 smlv) &, $0233451^{* \star *}$ & ,0067882 &, $0096197^{* * *}$ \\
\hline Cantidad de personas en el hogar & ,0157342 &,- 0056101 &,- 0105994 \\
\hline Subsidio &, 1740022 &, $3564096^{* * *}$ & ,2776599* \\
\hline Presencia del padre en el hogar & ,0254503 &,- 0027923 &,- 0058945 \\
\hline Presencia de la madre en el hogar &,- 1019589 &,- 0163496 &,- 0081669 \\
\hline Etnia &,- 0588023 &,- 0036955 &,- 0166654 \\
\hline $\begin{array}{l}\text { Nivel educativo máximo alcanzado } \\
\text { por la madre (universitaria) }\end{array}$ & ,0182434 &,- 0017984 &,- 0015428 \\
\hline \multirow[t]{2}{*}{$\begin{array}{l}\text { Nivel educativo máximo alcanzado } \\
\text { por el padre (universitaria) }\end{array}$} &,- 0884514 & ,0073777 &,- 0010305 \\
\hline & $\begin{array}{l}\text { Núm. obs. } 729 \\
\text { Chi 2: } 49,98 \\
\text { Prob>chi2: } 0,0000 \\
\text { Pseudo R2: } 0,0524 \\
\text { Estat classification- } \\
\text { correctly classified: } \\
64,75 \%\end{array}$ & $\begin{array}{l}\text { Núm. obs. } 1134 \\
\text { Chi 2: } 51,92 \\
\text { Prob>chi2: } 0,0000 \\
\text { Pseudo R2: } 0,0347 \\
\text { Estat classification- } \\
\text { correctly classified: } \\
64,20 \%\end{array}$ & $\begin{array}{l}\text { Núm. obs. } 1234 \\
\text { Chi 2: } 70,75 \\
\text { Prob>chi2: } 0,0000 \\
\text { Pseudo R2: } 0,0428 \\
\text { Estat classification- } \\
\text { correctly classified: } \\
63,94 \%\end{array}$ \\
\hline
\end{tabular}

Fuente: Construcción Propia.

El quinto perfil corresponde a los hogares con ingresos menores o iguales a $4 \mathrm{SMLV}$, en los cuales los padres alcanzaron un nivel educativo universitario. En este caso, al igual que en los demás, el ingreso y el 
GAPP. Nueva Época - N. 21, Mayo-Octubre 2019 - ISSN: 1989-8991 - DOI: 10.24965/gapp.v0i21.10522 - [Págs. 125-149]

Evaluación integral de la política pública de vivienda de interés social en Bogotá, 2008-2016

Mayra Alejandra Lozano Rodríguez / Hernán Darío Enríquez Sierra

subsidio son los principales determinantes de la tenencia de vivienda, dado que ante un aumento en el ingreso en 100.000 \$, la probabilidad incrementó en un 2,33\% y 1\%, en los años 2010 y 2015 , respectivamente.

Igualmente, ser beneficiario del subsidio de vivienda aumentó la probabilidad de tenencia en un $35,64 \%$ y $27,76 \%$, durante los años 2012 y 2015 , respecto a los hogares que no recibieron subsidio.

Perfil 6: Hogares con ingresos no superiores a 2 salarios mínimos legales y nivel máximo educativo alcanzado por los padres «superior».

TABLA 10. MODELO DE PROBABILIDAD DE TENENCIA DE VIVIENDA, DADAS LAS CARACTERÍSTICAS DE LOS HOGARES CON EL PERFIL 5

\begin{tabular}{|c|c|c|c|}
\hline Variable & $\begin{array}{l}\text { Cambios marginales } \\
2010\end{array}$ & $\begin{array}{l}\text { Cambios marginales } \\
2012\end{array}$ & $\begin{array}{c}\text { Cambios marginales } \\
2015\end{array}$ \\
\hline Ingreso del hogar (2 smlv) &, $0234769^{* * *}$ &, $0123121^{*}$ &, $0165508^{* * *}$ \\
\hline Cantidad de personas en el hogar & 0158231 &,- 0101753 &,- 0182365 \\
\hline Subsidio & , 1473226 &, $4554169^{* * *}$ &, $3477815^{\star *}$ \\
\hline Presencia del padre en el hogar & ,0249099 &,- 0050811 &,- 0102226 \\
\hline Presencia de la madre en el hogar &,$- 1152103^{*}$ &,- 0302355 &,- 0142074 \\
\hline Etnia &,- 0631408 &,- 0067318 &,- 0293309 \\
\hline $\begin{array}{l}\text { Nivel educativo máximo alcanzado } \\
\text { por la madre (universitaria) }\end{array}$ & 0179922 &,- 0032687 &,- 0026599 \\
\hline \multirow[t]{2}{*}{$\begin{array}{l}\text { Nivel educativo máximo alcanzado } \\
\text { por el padre (universitaria) }\end{array}$} &,- 983367 & ,0132659 &,- 0017754 \\
\hline & $\begin{array}{l}\text { Núm. obs. } 729 \\
\text { Chi 2: } 49,98 \\
\text { Prob>chi2: } 0,0000 \\
\text { Pseudo R2: } 0,0524 \\
\text { Estat classification- } \\
\text { correctly classified: } \\
64,75 \%\end{array}$ & $\begin{array}{l}\text { Núm. obs. } 1134 \\
\text { Chi 2: } 51,92 \\
\text { Prob>chi2: } 0,0000 \\
\text { Pseudo R2: } 0,0347 \\
\text { Estat classification- } \\
\text { correctly classified: } \\
64,20 \%\end{array}$ & $\begin{array}{l}\text { Núm. obs. } 1234 \\
\text { Chi 2: } 70,75 \\
\text { Prob>chi2: } 0,0000 \\
\text { Pseudo R2: } 0,0428 \\
\text { Estat classification- } \\
\text { correctly classified: } \\
63,94 \%\end{array}$ \\
\hline
\end{tabular}

${ }^{*}$ Significativas al $10 \% .{ }^{* *}$ Significancia al $5 \% .{ }^{* * *}$ Significancia al $1 \%$.

Fuente: Construcción Propia.

El sexto perfil corresponde a los hogares con ingresos menores o iguales a $2 \mathrm{SMLV}$, en los cuales los padres alcanzaron un nivel educativo universitario. Nuevamente, el ingreso y el subsidio son los principales determinantes de la tenencia de vivienda, dado que ante un aumento en el ingreso en 100.000 \$, la probabilidad incrementó en un $2 \%$ en los años 2010 y 2015, y 1\% en el año 2012.

Igualmente, ser beneficiario del subsidio de vivienda aumentó la probabilidad de tenencia en un $45 \%$ y $35 \%$, durante los años 2012 y 2015 , respecto a los hogares que no recibieron subsidio.

\section{Acerca de los resultados}

La mayoría de las variables que representan las condiciones de vulnerabilidad no arrojaron resultados significativos; sin embargo en los diferentes perfiles establecidos, dos de ellas obtuvieron el nivel de significancia que sustenta que el modelo no tiene problemas en su formulación.

Los principales determinantes para la adquisición de vivienda son el ingreso y el subsidio; no se hallaron diferencias relevantes en la probabilidad de tenencia en ninguno de los casos establecidos, según los perfiles de hogares diseñados para la aplicación del modelo logit.

Dado que el desembolso del subsidio está supeditado a la aprobación del crédito hipotecario, y la aprobación del crédito al ingreso del hogar y la capacidad de endeudamiento, esta relación es evidente. De esta 
GAPP. Nueva Época - N. 21, Mayo-Octubre 2019 - ISSN: 1989-8991 - DOI: 10.24965/gapp.v0i21.10522 - [Págs. 125-149]

Evaluación integral de la política pública de vivienda de interés social en Bogotá, 2008-2016

Mayra Alejandra Lozano Rodríguez / Hernán Darío Enríquez Sierra

manera, se identificó que poseer las condiciones de vulnerabilidad establecidas en el modelo, no garantiza una mayor probabilidad en la tenencia de vivienda; de allí que entre el año 2009 y 2012 sólo se haya efectuado una asignación de subsidios para VIP, correspondiente al 10,24\% de la producción total de VIS.

\subsubsection{Análisis de aspectos de calidad de vida no inherentes a la política}

Teniendo en cuenta que la normatividad vigente contempla el cumplimiento de requerimientos básicos para el desarrollo de proyectos de vivienda de interés social, que inciden en la calidad de vida (materiales de construcción utilizados, la provisión de servicios públicos, y la generación de un entorno social que garantice la existencia de colegios, puestos de salud, parques entre otros), este espacio presenta una reflexión sobre aspectos adicionales de calidad vida, que reflejan problemáticas de los hogares beneficiarios de la política, con relación a la demás población que cuenta con características similares y no ha sido beneficiada.

Se utilizaron los resultados de la encuesta de calidad de vida del DANE, aplicada en tres años diferentes $\left(2010,2012\right.$ y 2015) y el factor de expansión ${ }^{10}$ para hacer inferencias sobre el número total de hogares que tienen un ingreso menor a 4 salarios mínimos legales vigentes, diferenciando a aquellos que han sido beneficiarios de la política durante los dos años anteriores a la aplicación de la encuesta, y aquellos que no lo han sido.

- Ruidos provenientes del exterior.

TABLA 11. DISTRIBUCIÓN: RUIDOS PROVENIENTES DEL EXTERIOR EN LOS HOGARES OBJETO DE POLÍTICA (CON INGRESOS DE HASTA 4 SMLV), BENEFICIARIOS Y NO BENEFICIARIOS DE SUBSIDIO

\begin{tabular}{ccccc}
\hline & \multicolumn{2}{c}{ SI } & \multicolumn{2}{c}{ NO } \\
\cline { 2 - 5 } & $\begin{array}{c}\text { Hogares } \\
\text { beneficiarios } \\
\text { de subsidio }\end{array}$ & $\begin{array}{c}\text { Hogares } \\
\text { no beneficiarios }\end{array}$ & $\begin{array}{c}\text { Hogares } \\
\text { beneficiarios } \\
\text { de subsidio }\end{array}$ & $\begin{array}{c}\text { Hogares } \\
\text { noneficiarios }\end{array}$ \\
\hline $\mathbf{2 0 1 5}$ & 13,95 & 30,86 & 86,05 & 69,14 \\
\hline $\mathbf{2 0 1 2}$ & 16,18 & 32,03 & 83,82 & 67,97 \\
\hline $\mathbf{2 0 1 0}$ & 0 & 29,17 & 100 & 70,83 \\
\hline
\end{tabular}

Fuente: Elaboración propia con base en la encuesta de calidad de vida, DANE.

Al preguntarle a los hogares sobre la existencia de ruidos en el exterior, se evidencia que la problemática se presenta con una menor frecuencia en quienes han sido beneficiarios de la política, razón por la cual no se puede atribuir este aspecto a la ubicación de los proyectos de vivienda de interés social, ni a ninguna de sus características, tales como las condiciones de habitabilidad o situaciones externas que generan efectos negativos en la calidad de vida.

- Malos olores procedentes del exterior.

TABLA 12. DISTRIBUCIÓN: MALOS OLORES PROCEDENTES DEL EXTERIOR EN LOS HOGARES OBJETO DE POLÍTICA (CON INGRESOS DE HASTA 4 SMLV), BENEFICIARIOS Y NO BENEFICIARIOS DE SUBSIDIO

\begin{tabular}{ccccc}
\hline & \multicolumn{2}{c}{ SI } & \multicolumn{2}{c}{ NO } \\
\cline { 2 - 5 } Año & $\begin{array}{c}\text { Hogares } \\
\text { beneficiarios } \\
\text { de subsidio }\end{array}$ & $\begin{array}{c}\text { Hogares } \\
\text { no beneficiarios }\end{array}$ & $\begin{array}{c}\text { Hogares } \\
\text { beneficiarios } \\
\text { de subsidio }\end{array}$ & $\begin{array}{c}\text { Hogares } \\
\text { beneficiarios }\end{array}$ \\
\hline $\mathbf{2 0 1 5}$ & 42,04 & 27,19 & 57,96 & 72,81 \\
\hline $\mathbf{2 0 1 2}$ & 39,91 & 27,70 & 60,09 & 72,30 \\
\hline $\mathbf{2 0 1 0}$ & 72,66 & 24,65 & 27,34 & 75,35 \\
\hline
\end{tabular}

Fuente: Elaboración propia con base en la encuesta de calidad de vida, DANE.

10 Al aplicar el factor de expansión, los resultados evidencian la apreciación de 6285 hogares beneficiarios de subsidio y 1.298.261 hogares no beneficiarios en el año 2010; 13.159 hogares beneficiados y 1.445 .468 no beneficiados en el año 2012 ; y 15.272 beneficiarios y 1.567.559 no beneficiarios, en el año 2015 . 
En cuanto a sí los hogares tienen problemas relacionados con la existencia de malos olores procedentes del exterior, se identifica una mayor frecuencia -casi en doble proporción- en los hogares beneficiarios de la política. Dado que en los últimos años, la construcción de vivienda de interés social se ha realizado principalmente en lugares periféricos, este puede ser considerado un aspecto particular que debe ser estudiado para el desarrollo de nuevas construcciones.

- Presencia de Basuras en las calles.

TABLA 13. Distribución: PRESENCIA de basuRAs EN LAS CALLES EN LOS HOGARES OBJeto de POLÍTICA (CON INGRESOS DE HASTA 4 SMLV), BENEFICIARIOS Y NO BENEFICIARIOS DE SUBSIDIO

\begin{tabular}{ccccc}
\hline & \multicolumn{2}{c}{ SI } & \multicolumn{2}{c}{ NO } \\
\cline { 2 - 5 } Año & $\begin{array}{c}\text { Hogares } \\
\text { beneficiarios } \\
\text { de subsidio }\end{array}$ & $\begin{array}{c}\text { Hogares } \\
\text { no beneficiarios }\end{array}$ & $\begin{array}{c}\text { Hogares } \\
\text { beneficiarios } \\
\text { de subsidio }\end{array}$ & $\begin{array}{c}\text { Hogares } \\
\text { no beneficiarios }\end{array}$ \\
\hline $\mathbf{2 0 1 5}$ & 44,49 & 33,66 & 55,51 & 66,34 \\
\hline $\mathbf{2 0 1 2}$ & 51,63 & 33,54 & 48,37 & 66,46 \\
\hline $\mathbf{2 0 1 0}$ & 0 & 24,88 & 100 & 75,12 \\
\hline
\end{tabular}

Fuente: Elaboración propia con base en la encuesta de calidad de vida, DANE.

Al igual que en el caso anterior, la presencia de basura en las calles es mencionada con mayor frecuencia en los hogares beneficiarios. Esta problemática puede presentarse debido a la ausencia de cultura ambiental por parte de la comunidad o a la falta de infraestructura o de implementación del sistema de recolección de desechos que el Estado debe garantizar.

- Contaminación en el aire.

TABLA 14. DISTRIBUCIÓN: CONTAMINACIÓN EN EL AIRE EN LOS HOGARES OBJETO DE POLÍtICA (CON INGRESOS DE HASTA 4 SMLV), BENEFICIARIOS Y NO BENEFICIARIOS DE SUBSIDIO

\begin{tabular}{ccccc}
\hline & \multicolumn{2}{c}{ SI } & No \\
\cline { 2 - 5 } Año & $\begin{array}{c}\text { Hogares } \\
\text { beneficiarios } \\
\text { de subsidio }\end{array}$ & $\begin{array}{c}\text { Hogares } \\
\text { no beneficiarios }\end{array}$ & $\begin{array}{c}\text { Hogares } \\
\text { beneficiarios } \\
\text { de subsidio }\end{array}$ & $\begin{array}{c}\text { Hogares } \\
\text { no beneficiarios }\end{array}$ \\
\hline $\mathbf{2 0 1 5}$ & 35,95 & 24,64 & 64,05 & 75,36 \\
\hline $\mathbf{2 0 1 2}$ & 32,84 & 25,31 & 67,16 & 74,69 \\
\hline
\end{tabular}

Fuente: Elaboración propia con base en la encuesta de calidad de vida, DANE.

La contaminación en el aire es un aspecto, que sí bien es mencionado con una mayor frecuencia en los hogares beneficiarios, no presenta una diferencia significativa con respecto a la población no beneficiaria, a excepción del año 2010.

- Invasión del espacio público. 
GAPP. Nueva Época - N. 21, Mayo-Octubre 2019 - ISSN: 1989-8991 - DOI: 10.24965/gapp.v0i21.10522 - [Págs. 125-149]

Evaluación integral de la política pública de vivienda de interés social en Bogotá, 2008-2016

Mayra Alejandra Lozano Rodríguez / Hernán Darío Enríquez Sierra

TABLA 15. DISTRIBUCIÓN: INVASIÓN DEL ESPACIO PÚBLICO EN LOS HOGARES OBJETO DE POLÍTICA (CON INGRESOS DE HASTA 4 SMLV), BENEFICIARIOS Y NO BENEFICIARIOS DE SUBSIDIO

\begin{tabular}{ccccc}
\hline & \multicolumn{2}{c}{ SI } & NO \\
\cline { 2 - 5 } Año & $\begin{array}{c}\text { Hogares } \\
\text { beneficiarios } \\
\text { de subsidio }\end{array}$ & $\begin{array}{c}\text { Hogares } \\
\text { no beneficiarios }\end{array}$ & $\begin{array}{c}\text { Hogares } \\
\text { beneficiarios } \\
\text { de subsidio }\end{array}$ & $\begin{array}{c}\text { Hogares } \\
\text { no beneficiarios }\end{array}$ \\
\hline $\mathbf{2 0 1 5}$ & 0 & 13,35 & 100 & 86,65 \\
\hline $\mathbf{2 0 1 2}$ & 0 & 13,39 & 100 & 86,61 \\
\hline $\mathbf{2 0 1 0}$ & 0 & 11,29 & 100 & 88,71 \\
\hline
\end{tabular}

Fuente: Elaboración propia con base en la encuesta de calidad de vida, DANE.

Los hogares beneficiarios expresaron no tener inconvenientes con la invasión del espacio público.

- Presencia de animales que causan molestias.

TABLA 16. DISTRIBUCIÓN: PRESENCIA DE ANIMALES QUE CAUSAN MOLESTIAS EN LOS HOGARES OBJETO DE POLÍTICA (CON INGRESOS DE HASTA 4 SMLV), BENEFICIARIOS Y NO BENEFICIARIOS DE SUBSIDIO

\begin{tabular}{cccccc}
\hline & \multicolumn{2}{c}{ SI } & \multicolumn{2}{c}{ NO } \\
\cline { 2 - 5 } Año & $\begin{array}{c}\text { Hogares } \\
\text { beneficiarios } \\
\text { de subsidio }\end{array}$ & $\begin{array}{c}\text { Hogares } \\
\text { no beneficiarios }\end{array}$ & $\begin{array}{c}\text { Hogares } \\
\text { beneficiarios } \\
\text { de subsidio }\end{array}$ & $\begin{array}{c}\text { Hogares } \\
\text { no beneficiarios }\end{array}$ \\
\hline $\mathbf{2 0 1 5}$ & 11,08 & 22,07 & 88,92 & 77,93 \\
\hline $\mathbf{2 0 1 2}$ & 12,86 & 22,39 & 87,14 & 77,61 \\
\hline $\mathbf{2 0 1 0}$ & 47,64 & 22,52 & 52,36 & 77,48 \\
\hline
\end{tabular}

Fuente: Elaboración propia con base en la encuesta de calidad de vida, DANE.

En cuanto a la presencia de animales que causan molestias, los hogares beneficiarios de la política manifiestan enfrentarse a esta problemática en una menor frecuencia con relación a los que no. Igualmente, se puede apreciar una diferencia considerable en el año 2010.

- Presencia de insectos, roedores, etc.

TABLA 17. DISTRIBUCIÓN: PRESENCIA DE INSECTOS, ROEDORES, ETC., EN LOS HOGARES OBJETO DE POLÍTICA (CON INGRESOS DE HASTA 4 SMLV), BENEFICIARIOS Y NO BENEFICIARIOS DE SUBSIDIO

\begin{tabular}{ccccc}
\hline & \multicolumn{2}{c}{ SI } & NO \\
\cline { 2 - 5 } Año & $\begin{array}{c}\text { Hogares } \\
\text { beneficiarios } \\
\text { de subsidio }\end{array}$ & $\begin{array}{c}\text { Hogares } \\
\text { no beneficiarios }\end{array}$ & $\begin{array}{c}\text { Hogares } \\
\text { beneficiarios } \\
\text { de subsidio }\end{array}$ & $\begin{array}{c}\text { Hogares } \\
\text { no beneficiarios }\end{array}$ \\
\hline $\mathbf{2 0 1 5}$ & 66,08 & 25,27 & 33,92 & 74,73 \\
\hline $\mathbf{2 0 1 2}$ & 67,80 & 26,12 & 32,20 & 73,88 \\
\hline $\mathbf{2 0 1 0}$ & 74,98 & 33,95 & 25,02 & 66,05 \\
\hline
\end{tabular}

Fuente: Elaboración propia con base en la encuesta de calidad de vida, DANE.

La presencia de insectos, roedores, entre otras plagas, es identificada como un problemática relevante en la población beneficiaria de la política; mientras que para esta población la frecuencia oscila entre un 66 y un $74 \%$, para los hogares no beneficiarios se presenta entre un 25 y un $34 \%$, respectivamente. 
GAPP. Nueva Época - N. 21, Mayo-Octubre 2019 - ISSN: 1989-8991 - DOI: 10.24965/gapp.v0i21.10522 - [Págs. 125-149]

Evaluación integral de la política pública de vivienda de interés social en Bogotá, 2008-2016

Mayra Alejandra Lozano Rodríguez / Hernán Darío Enríquez Sierra

- Atracos o robos durante los últimos 12 meses.

TABLA 18. DISTRIBUCIÓN: ATRACOS O ROBOS EN LOS HOGARES OBJETO DE POLÍTICA (CON INGRESOS DE HASTA 4 SMLV), BENEFICIARIOS Y NO BENEFICIARIOS DE SUBSIDIO

\begin{tabular}{cccccc}
\hline & \multicolumn{2}{c}{ SI } & \multicolumn{2}{c}{ NO } & \\
\cline { 2 - 5 } Año & $\begin{array}{c}\text { Hogares } \\
\text { beneficiarios } \\
\text { de subsidio }\end{array}$ & $\begin{array}{c}\text { Hogares } \\
\text { no beneficiarios }\end{array}$ & $\begin{array}{c}\text { Hogares } \\
\text { beneficiarios } \\
\text { de subsidio }\end{array}$ & $\begin{array}{c}\text { Hogares } \\
\text { no beneficiarios }\end{array}$ \\
\hline $\mathbf{2 0 1 5}$ & 45,09 & 23,91 & 54,91 & 76,09 \\
\hline $\mathbf{2 0 1 2}$ & 52,33 & 24,19 & 47,67 & 75,81 \\
\hline $\mathbf{2 0 1 0}$ & 47,64 & 23,90 & 52,36 & 76,10 \\
\hline
\end{tabular}

Fuente: Elaboración propia con base en la encuesta de calidad de vida, DANE.

Otro suceso que se presenta con mayor frecuencia en los hogares beneficiarios de la política, es haber sido víctima de atracos o robos. Aproximadamente la mitad de la población indicó, durante los tres años, sufrir un episodio de este tipo; mientras que tan sólo un cuarto de la población que no ha sido beneficiaria, evidenció esta problemática.

- Otro hecho violento (homicidios, asesinatos, secuestros, lesiones personales, violaciones, extorsiones y desalojos) durante los últimos 12 meses.

TABLA 19. DISTRIBUCIÓN: OTRO HECHO VIOLENTO EN LOS HOGARES OBJETO DE POLÍTICA (CON INGRESOS DE HASTA 4 SMLV), BENEFICIARIOS Y NO BENEFICIARIOS DE SUBSIDIO

\begin{tabular}{ccccc}
\hline & \multicolumn{2}{c}{ SI } & NO \\
\cline { 2 - 5 } Año & $\begin{array}{c}\text { Hogares } \\
\text { beneficiarios } \\
\text { de subsidio }\end{array}$ & $\begin{array}{c}\text { Hogares } \\
\text { no beneficiarios }\end{array}$ & $\begin{array}{c}\text { Hogares } \\
\text { beneficiarios } \\
\text { de subsidio }\end{array}$ & $\begin{array}{c}\text { Hogares } \\
\text { no beneficiarios }\end{array}$ \\
\hline $\mathbf{2 0 1 5}$ & 33,43 & 8,37 & 66,57 & 91,63 \\
\hline $\mathbf{2 0 1 2}$ & 38,80 & 8,83 & 61,20 & 91,17 \\
\hline $\mathbf{2 0 1 0}$ & 0 & 1,49 & 100 & 98,51 \\
\hline
\end{tabular}

Fuente: Elaboración propia con base en la encuesta de calidad de vida, DANE.

Los hogares beneficiarios indicaron que durante el último año fueron víctimas de episodios violentos. La frecuencia en la que se presenta este problema es más alta con relación al otro grupo poblacional.

\subsubsection{Percepción del nivel de la calidad de vida}

En coherencia con el planteamiento teórico sobre el bienestar que hace Currie, este apartado tiene como fin identificar el nivel de satisfacción de los hogares con relación a sus condiciones de vida, partiendo de la correlación existente entre el desarrollo económico y la provisión de necesidades no pecuniarias, que en su conjunto promueven la emisión de juicios de los individuos sobre su bienestar.

Al igual en los anteriores apartados, se toman los resultados de las encuesta de calidad de vida (2010, 2012 y 2015), así:

- ¿Cómo se siente en el barrio donde vive? 
GAPP. Nueva Época - N. 21, Mayo-Octubre 2019 - ISSN: 1989-8991 - DOI: 10.24965/gapp.v0i21.10522 - [Págs. 125-149]

Evaluación integral de la política pública de vivienda de interés social en Bogotá, 2008-2016 Mayra Alejandra Lozano Rodríguez / Hernán Darío Enríquez Sierra

TABLA 20. PERCEPCIÓN DE LOS HOGARES CON INGRESOS MENORES A 4 SMLV SOBRE EL BARRIO DONDE VIVEN

\begin{tabular}{ccccc}
\hline & \multicolumn{2}{c}{ Seguro } & \multicolumn{2}{c}{ Inseguro } \\
\cline { 2 - 5 } Año & $\begin{array}{c}\text { Hogares } \\
\text { beneficiarios } \\
\text { de subsidio }\end{array}$ & $\begin{array}{c}\text { Hogares } \\
\text { no beneficiarios }\end{array}$ & $\begin{array}{c}\text { Hogares } \\
\text { beneficiarios } \\
\text { de subsidio }\end{array}$ & $\begin{array}{c}\text { Hogares } \\
\text { neneficiarios }\end{array}$ \\
\hline $\mathbf{2 0 1 5}$ & 42,24 & 56,80 & 57,76 & 43,20 \\
\hline $\mathbf{2 0 1 2}$ & 40,13 & 56,17 & 59,87 & 43,83 \\
\hline $\mathbf{2 0 1 0}$ & 56,17 & 52,68 & 43,83 & 47,32 \\
\hline
\end{tabular}

Fuente: Elaboración propia con base en la encuesta de calidad de vida, DANE.

Los hogares beneficiarios de subsidio expresan sentirse inseguros con una mayor frecuencia que los hogares que no lo han recibido (o por lo menos recientemente); la diferencia entre los dos grupos poblacionales durante los últimos años oscila en alrededor de un 15\%. Si bien, los temas de seguridad deben ingresar en la agenda, dados los alcances de este estudio, no podría asegurarse que este aspecto se presente con mayor frecuencia en los hogares con 4 smlv, frente al resto de la población.

- ¿Actualmente las condiciones de su hogar son?

TABLA 21. PERCEPCIÓN DE LOS HOGARES CON INGRESOS MENORES A 4 SMLV SOBRE LAS CONDICIONES DEL HOGAR

\begin{tabular}{cccccccccc}
\hline & \multicolumn{2}{c}{ Muy buenas } & \multicolumn{2}{c}{ Buenas } & \multicolumn{2}{c}{ Regulares } & \multicolumn{2}{c}{ Malas } \\
\cline { 2 - 10 } & $\begin{array}{c}\text { Hogares } \\
\text { beneficiarios } \\
\text { de subsidio }\end{array}$ & $\begin{array}{c}\text { Hogares } \\
\text { no }\end{array}$ & $\begin{array}{c}\text { Hogares } \\
\text { beneficiarios }\end{array}$ & $\begin{array}{c}\text { Hogares } \\
\text { de subsidio }\end{array}$ & $\begin{array}{c}\text { Hogares } \\
\text { beneficiarios } \\
\text { beneficiarios }\end{array}$ & $\begin{array}{c}\text { Hogares } \\
\text { no }\end{array}$ & $\begin{array}{c}\text { Hogares } \\
\text { beneficiarios } \\
\text { de subsidio }\end{array}$ & $\begin{array}{c}\text { Hogares } \\
\text { no } \\
\text { beneficiarios } \\
\text { de subsidio }\end{array}$ \\
\hline $\mathbf{2 0 1 5}$ & 0 & 7,75 & 39,44 & 66,27 & 60,56 & 24,40 & 0 & 1,58 \\
\hline $\mathbf{2 0 1 2}$ & 0 & 6,97 & 50,32 & 1,73 & 49,68 & 24,97 & 0 & 1,73 \\
\hline $\mathbf{2 0 1 0}$ & 27,34 & 5,73 & 47,64 & 68,98 & 25,02 & 28,76 & 0 & 2,26 \\
\hline
\end{tabular}

Fuente: Elaboración propia con base en la encuesta de calidad de vida, DANE.

En cuanto a las condiciones de los hogares, se evidencian diferencias significativas entre los dos grupos poblacionales. La percepción en aquellos que han sido beneficiarios de la política ha ido focalizándose y aumentando en el concepto «regular», pasando de una frecuencia del $25 \%$ en el 2010 al 60,56\% en el 2015. Las razones por las cuales los hogares realizan este juicio son desconocidas, por lo cual la indagación sobre este aspecto será uno de los retos de la política.

- ¿Con relación al hogar donde usted se crió, este hogar vive económicamente?

TABLA 22. PERCEPCIÓN DE LOS HOGARES CON INGRESOS MENORES A 4 SMLV SOBRE LAS CONDICIONES ECONÓMICAS DEL HOGAR

\begin{tabular}{ccccccc}
\hline & \multicolumn{2}{c}{ Mejor } & \multicolumn{2}{c}{ Igual } & \multicolumn{2}{c}{ Peor } \\
\cline { 2 - 7 } Año & $\begin{array}{c}\text { Hogares } \\
\text { beneficiarios } \\
\text { de subsidio }\end{array}$ & $\begin{array}{c}\text { Hogares } \\
\text { no } \\
\text { beneficiarios }\end{array}$ & $\begin{array}{c}\text { Hogares } \\
\text { beneficiarios } \\
\text { de subsidio }\end{array}$ & $\begin{array}{c}\text { Hogares } \\
\text { no } \\
\text { beneficiarios }\end{array}$ & $\begin{array}{c}\text { Hogares } \\
\text { beneficiarios } \\
\text { de subsidio }\end{array}$ & $\begin{array}{c}\text { Hogares } \\
\text { no } \\
\text { beneficiarios }\end{array}$ \\
\hline $\mathbf{2 0 1 5}$ & 66,36 & 52,28 & 13,95 & 37,31 & 19,69 & 1,41 \\
\hline $\mathbf{2 0 1 2}$ & 72,67 & 50,91 & 16,18 & 38,03 & 11,14 & 11,05 \\
\hline $\mathbf{2 0 1 0}$ & 53,84 & 51,39 & 27,34 & 34,55 & 18,81 & 14,05 \\
\hline
\end{tabular}

Fuente: Elaboración propia con base en la encuesta de calidad de vida, DANE. 
Los hogares beneficiarios consideran, en su mayoría, que viven mejor económicamente respecto al hogar de crianza; esto puede ser un indicador de movilidad social que evidencia la capacidad que tienen sus miembros para incrementar sus ingresos. Es también un factor considerable, el hecho de que su percepción es mucho más favorable, que la de aquellos que no han sido beneficiarios de subsidios.

- ¿Usted piensa que el nivel de vida actual de este hogar, respecto al que tenía es?

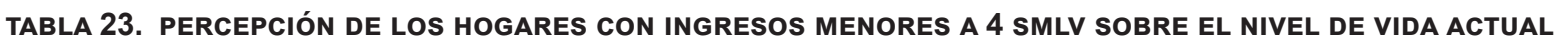

\begin{tabular}{ccccccc}
\hline & \multicolumn{2}{c}{ Mejor } & \multicolumn{2}{c}{ Igual } & & Peor \\
\cline { 2 - 7 } & $\begin{array}{c}\text { Hogares } \\
\text { beneficiarios } \\
\text { de subsidio }\end{array}$ & $\begin{array}{c}\text { Hogares } \\
\text { no } \\
\text { beneficiarios }\end{array}$ & $\begin{array}{c}\text { Hogares } \\
\text { beneficiarios } \\
\text { de subsidio }\end{array}$ & $\begin{array}{c}\text { Hogares } \\
\text { no } \\
\text { beneficiarios }\end{array}$ & $\begin{array}{c}\text { Hogares } \\
\text { beneficiarios } \\
\text { de subsidio }\end{array}$ & $\begin{array}{c}\text { Hogares } \\
\text { no } \\
\text { beneficiarios }\end{array}$ \\
\hline $\mathbf{2 0 1 5}$ & 8,41 & 45,81 & 79,93 & 42,80 & 11,66 & 11,39 \\
\hline $\mathbf{2 0 1 2}$ & 21,47 & 44,88 & 65,00 & 43,43 & 13,53 & 11,69 \\
\hline $\mathbf{2 0 1 0}$ & 18,81 & 48,10 & 52,36 & 35,31 & 28,83 & 16,59 \\
\hline
\end{tabular}

Fuente: Elaboración propia con base en la encuesta de calidad de vida, DANE.

La mayoría de los hogares beneficiarios indican que no existen cambios sustanciales en su nivel de vida. Esta percepción ha ido aumentando paulatinamente, presentándose una frecuencia del $80 \%$ en el 2015. Por el contrario, la mayoría de los hogares que no han sido beneficiarios consideran que su nivel de vida es mejor, razón por la cual se puede inferir que la percepción de los beneficiarios denota una característica particular para este grupo poblacional, la cual deberá ser estudiada.

- ¿Los ingresos de su hogar?

TABLA 24. PERCEPCIÓN DE LOS HOGARES CON INGRESOS MENORES A 4 SMLV SOBRE LA SUFICIENCIA DE LOS INGRESOS

\begin{tabular}{|c|c|c|c|c|c|c|}
\hline \multirow[b]{2}{*}{ Año } & \multicolumn{2}{|c|}{$\begin{array}{c}\text { No alcanzan para cubrir los } \\
\text { gastos mínimos }\end{array}$} & \multicolumn{2}{|c|}{$\begin{array}{l}\text { Sólo alcanzan para cubrir } \\
\text { los gastos mínimos }\end{array}$} & \multicolumn{2}{|c|}{$\begin{array}{c}\text { Cubren más de los gastos } \\
\text { mínimos }\end{array}$} \\
\hline & $\begin{array}{c}\text { Hogares } \\
\text { beneficiarios } \\
\text { de subsidio }\end{array}$ & $\begin{array}{c}\text { Hogares } \\
\text { no } \\
\text { beneficiarios }\end{array}$ & $\begin{array}{c}\text { Hogares } \\
\text { beneficiarios } \\
\text { de subsidio }\end{array}$ & $\begin{array}{c}\text { Hogares } \\
\text { no } \\
\text { beneficiarios }\end{array}$ & $\begin{array}{c}\text { Hogares } \\
\text { beneficiarios } \\
\text { de subsidio }\end{array}$ & $\begin{array}{c}\text { Hogares } \\
\text { no } \\
\text { beneficiarios }\end{array}$ \\
\hline 2015 & 17,39 & 21,80 & 82,61 & 68,79 & 0 & 9,41 \\
\hline 2012 & 20,18 & 22,42 & 79,82 & 68,48 & 0 & 9,10 \\
\hline 2010 & 72,66 & 36,31 & 27,34 & 58,31 & 0 & 5,38 \\
\hline
\end{tabular}

Fuente: Elaboración propia con base en la encuesta de calidad de vida, DANE.

La percepción de los hogares beneficiarios indica que los ingresos sólo alcanzan para cubrir los gastos mínimos del hogar. La frecuencia con la que los hogares manifiestan esta apreciación ha aumentado significativamente en los últimos años, pasando de $27,34 \%$ en el 2010 a $82,61 \%$ en el 2015 . Al comparar los dos grupos poblaciones, se identifica nuevamente que el nivel de satisfacción en los hogares benefeciarios, con relación a este aspecto, es menor.

- ¿Usted se considera pobre? 
GAPP. Nueva Época - N. 21, Mayo-Octubre 2019 - ISSN: 1989-8991 - DOI: 10.24965/gapp.v0i21.10522 - [Págs. 125-149]

Evaluación integral de la política pública de vivienda de interés social en Bogotá, 2008-2016 Mayra Alejandra Lozano Rodríguez / Hernán Darío Enríquez Sierra

TABLA 25. PERCEPCIÓN DE LOS HOGARES CON INGRESOS MENORES A 4 SMLV SOBRE LA CONDICIÓN DE POBREZA

\begin{tabular}{cccccc}
\hline & \multicolumn{2}{c}{ SI } & \multicolumn{2}{c}{ NO } \\
\cline { 2 - 5 } Año & $\begin{array}{c}\text { Hogares } \\
\text { beneficiarios } \\
\text { de subsidio }\end{array}$ & $\begin{array}{c}\text { Hogares } \\
\text { no beneficiarios }\end{array}$ & $\begin{array}{c}\text { Hogares } \\
\text { beneficiarios } \\
\text { de subsidio }\end{array}$ & $\begin{array}{c}\text { Hogares } \\
\text { no beneficiarios }\end{array}$ \\
\hline $\mathbf{2 0 1 5}$ & 38,39 & 28,49 & 61,61 & 71,51 \\
\hline $\mathbf{2 0 1 2}$ & 44,55 & 29,98 & 55,45 & 70,02 \\
\hline $\mathbf{2 0 1 0}$ & 47,64 & 42,39 & 52,36 & 57,61 \\
\hline
\end{tabular}

Fuente: Elaboración propia con base en la encuesta de calidad de vida, DANE.

Al consultarle a los hogares sí se consideran pobres, se identifica que nuevamente esta apreciación está asociada en una mayor frecuencia en los hogares beneficiarios de la política; sin embargo es de resaltar que dicha percepción ha disminuido a través del tiempo, pasando de $47,64 \%$ en el 2010 al 38,89\% en el año 2015.

\section{RESULTADOS DE LA EVALUACIÓN}

Las prácticas evaluativas han sido inherentes a la dinámica natural de los programas de vivienda social en Colombia, y han revelado persistentes problemas, como el déficit cualitativo y cuantitativo, la falta de asequibilidad de los hogares con menores recursos a las líneas de financiación, la pobreza y la falta de cobertura y focalización de la política; lo que sustenta la necesidad de realizar una aproximación hacia una «evaluación integral» que basándose en los postulados de Currie ${ }^{11}$, logre articular varias dimensiones: desarrollo, crecimiento económico y mejoramiento de las condiciones de calidad de vida de la población objeto de la política, tanto en términos cuantitativos como en su apreciación.

Ahora bien, como se mencionó anteriormente este ejercicio investigativo tuvo como propósito determinar el nivel de efectividad de la política de vivienda, mediante la valoración de la relación existente entre sus objetivos y los resultados alcanzados; en este sentido, es pertinente mencionar la perspectiva gubernamental en el marco del Plan Nacional de Desarrollo (2014-2018), que denotado por la incorporación del país a la OCDE, establece:

"contar con "mejores políticas para mejorar el bienestar de los ciudadanos". Esto significa que la gobernanza y las instituciones deben operar en función del mejoramiento de las múltiples dimensiones del bienestar de la población (salud, educación, empleo, vivienda, entre otros) y del desarrollo de los territorios mediante la adopción y el cumplimiento de estándares de calidad de gobierno» (pág. 590).

Nótese que bajo esta declaración, muy acorde a los postulados de Currie, las políticas implementadas deben necesariamente enfocarse en el mejoramiento de las dimensiones de bienestar, entre las cuales se encuentra la vivienda. De esta manera, el PND (2014-2018) estableció de forma específica la función del Estado en la garantía del acceso en igualdad de condiciones a servicios fundamentales como la vivienda, para toda la población (pág. 67), por lo cual esta promulgación se constituyó en la línea base para elaborar el juicio sobre el nivel de efectividad de la política.

En lo referente a crecimiento económico, la evaluación presentada en este documento evidenció que en términos de la producción total de vivienda, entre los años 2007 y 2011, se duplicó el número de unidades, pasando de 24.219 a 46.112; a partir de ese mismo año hasta el 2015, se ha fabricado un promedio anual de 47.180 unidades, de las cuales 15.802 son de interés social y 6.070 VIP. Aun así, la sostenibilidad presentada desde el año 2011 en el promedio de producción de unidades de vivienda, no ha sido significativamente representativa en el PIB, el porcentaje del sector de la construcción en éste ha oscilado entre el

11 Currie planteó la estimulación del sector de la construcción como mecanismo de jalonamiento de la economía, el mejoramiento infraestructural del país y el mejoramiento de las condiciones de bienestar de la población en condición de pobreza. 
$4,45 \%$ a $6,86 \%$, con tendencia a la baja desde el 2008 (De 6,44\% a 4,53\%). De la misma manera, el índice de ocupación en el sector ha fluctuado entre el $5 \%$ y el $7 \%$, no reflejando grandes cambios en la disminución del índice de desempleo.

Es de destacar que justamente a partir del año 2011 los precios de vivienda han incrementado hasta en un $40 \%$, y que lo han hecho en una mayor proporción con relación al incremento de los costos de producción. Este fenómeno puede incidir directamente en el indicador de déficit cuantitativo; sin embargo en Colombia la última medición se realizó en el año 2005, razón por la cual a futuro éste deberá ser un tema relevante para evidenciar la incidencia en la cobertura y capacidad de la política para generar transformaciones sociales a profundidad.

Ahora bien, entendiendo que la política establece como instrumento de acceso a la vivienda, el otorgamiento de subsidios a través de la aplicación de un modelo de vulnerabilidad, se revisó la incidencia de las características de dicho modelo en la tenencia de vivienda en los hogares con ingresos de hasta 4 SMLV, identificando que los principales determinantes para la adquisición de vivienda son el ingreso y el subsidio; no se hallaron diferencias relevantes en la probabilidad de tenencia en ninguno de los casos establecidos, según los perfiles de hogares diseñados para la aplicación del modelo logit.

Dado que el desembolso del subsidio está supeditado a la aprobación del crédito hipotecario, y la aprobación del crédito al ingreso del hogar y la capacidad de endeudamiento, esta relación es evidente. De esta manera, se identificó que poseer las condiciones de vulnerabilidad establecidas en el modelo, no garantiza una mayor probabilidad en la tenencia de vivienda; de allí que entre el año 2009 y 2012 sólo se haya efectuado una asignación de subsidios para VIP, correspondiente al 10,24\% de la producción total de VIS.

Por otra parte, partiendo de las apreciaciones de Currie sobre el bienestar y la incidencia que debe tener el crecimiento económico en las condiciones de vida de la población más pobre, respecto a los aspectos de calidad de vida evaluados, se identificó que varios de ellos se presentan con una frecuencia similar en los beneficiarios y no beneficiarios, tales como: ruidos provenientes en el exterior, contaminación en el aire, invasión del espacio público, presencia de animales que causas molestias, y hechos violentos; sin embargo hay aspectos que se presentan con mayor intensidad en los hogares que han sido beneficiarios de subsidio en los últimos 6 años, dentro de los cuales se encuentran malos olores procedentes del exterior, presencia de basura en las calles -que pueden relacionarse con la falta de cultura de la población o con un tema de incapacidad de la infraestructura para prestar el servicio de recolección de basuras-, así como la presencia de plagas (insectos, roedores, entre otros), y ser víctimas de atracos o robos.

Igualmente, al revisar el nivel de satisfacción de la población objeto de la política respecto a la calidad de vida, es interesante que tanto beneficiarios como no beneficiarios manifiestan en igual proporción, sentirse inseguros en el barrio donde viven y expresan vivir económicamente mejor que en el lugar de crianza; no obstante al preguntarles a los hogares beneficiarios respecto a sus condiciones de vida, la mayoría expresa que son regulares, con un incremento en la frecuencia de esta apreciación que va desde el $25 \%$ en el 2010 al $60 \%$ en el 2015 , en este sentido es necesario que se indaguen sobre las causas de la percepción, ya que si bien se evidencia un mejoramiento económico, no de las condiciones como tal, dando cabida a la pregunta ¿qué entiende la población por mejores condiciones de vida?.

En coherencia con lo anterior, al consultar a los hogares beneficiarios sobre el nivel de vida actual respecto al que tenía antes, la mayoría expresó que es igual, e incluso aumentó la apreciación, pasando del $52 \%$ en el 2010 al $80 \%$ en el 2015. Dado que los no beneficiarios expresan en su mayoría que el nivel actual es mejor, es necesario indagar sobre este aspecto con una mayor rigurosidad.

Por otra parte, si bien los hogares beneficiarios reconocen un mejoramiento de las condiciones económicas con respecto al hogar de crianza, también expresan que los ingresos sólo alcanzan para cubrir los gastos mínimos, del $27 \%$ en el 2010 al $82 \%$ en el 2015. En cuanto a sí los hogares se consideran pobres, aunque la percepción es mayor en los beneficiarios, es relevante mencionar que la frecuencia fue disminuyendo de un 47,64\% en el año 2010 a un 38,39 en el año 2015, mientras que en la población no beneficiaria pasó de un $42,39 \%$ a un $28,49 \%$.

En síntesis y a la luz del objetivo del PND 2014-2018, podemos afirmar que en términos de crecimiento económico, a partir del año 2012 el sector de la construcción ha logrado sostener una producción similar de unidades de vivienda, lo cual no ha implicado un incremento sustancial de la participación en el PIB, ni una incidencia significativa en el índice de desempleo. Así mismo se evidencia una sostenibilidad en la producción de vivienda de interés social, que de manera prioritaria debe ser contrastada con una nueva medición del déficit cuantitativo, y por otro lado, identificar el remanente de la producción que no está siendo comprada por la población objeto de la política, es decir aquellos que cuentan con ingresos no superiores a 4 SMLV, y así diseñar estrategias de focalización más efectivas. 
A su vez, teniendo en cuenta que las problemáticas asociadas al nivel de calidad de vida que se están presentando en los hogares beneficiarios -malos olores, presencia de basuras, presencia de plagas, e inseguridad-, pueden atribuirse a la ubicación de los proyectos de vivienda en las periferias, estos temas deben convertirse en aspectos prioritarios de estudio que conlleven a la promulgación de programas, que articulados a la política de vivienda, logren mejorar las condiciones de hábitat.

Finalmente, contemplando la apreciación del grupo poblacional beneficiario como factor de medición de bienestar, es de mencionar que si bien la mayoría reconocen contar con mejores condiciones económicas, no consideran que sus condiciones de vida hayan mejorado sustancialmente, razón por la cuál es necesario diseñar instrumentos que permitan conocer a fondo esta concepción, lo que podría develar aspectos o problemáticas, que contribuyan al ajuste o formulación de nuevas política o programas.

\section{REFERENCIAS BIBLIOGRÁFICAS}

AGUDELO, C., VACA, M. \& GARCÍA, C. (2013): "Modelo de producción social de hábitat frente al modelo de mercado en la construcción de vivienda de interés social", en Revista Tecnura, vol. 17, núm. 38, págs. 27-52. DOI: https:// doi.org/10.14483/udistrital.jour.tecnura.2013.4.a03.

BOUZA, A. (2000): "Reflexiones acerca del uso de los conceptos de eficiencia, eficacia y efectividad en el sector salud", en Revista Cubana de Salud Pública. Escuela Nacional de Salud Pública "Carlos J. Finlay», vol. 29, núm 1 , págs. 51-54.

CAMARGO, A. P. \& HURTADO, A. (2011): "Vivienda y pobreza: una relación compleja. Marco conceptual y caracterización de Bogotá", en Cuadernos de Vivienda y Urbanismo, vol. 4, núm. 8, págs. 224-246.

CASTRILLO, M. (2004): "Vivienda social y planificación urbanística: vestigios reformistas en la práctica actual", en Sociologia, Revista da Faculdade de Letras da Universidade do Porto (Porto, Portugal), núm. 13 (2003).

CHIAPPE, M. (1999): La política de vivienda de interés social en Colombia en los noventa. Santiago de Chile: Naciones Unidas.

ESCALLÓN, G. (2012): "La vivienda de interés social en Colombia, principios y retos", en Revista de Ingeniería, núm. 35, págs. 55-60. Universidad de los Andes.

FERNÁNDEZ, S. (2000): "La efectividad de los programas sociales. Enfoques y técnicas de la evaluación de procesos", en Revista de Psicología del Trabajo y de las Organizaciones, vol. 16, núm. 3, pág. 260.

FIQUE, L. F. (2005): "La habitabilidad de la vivienda de interés social en Colombia. Un enfoque en los procesos y las decisiones", en Revista INVI, vol. 20, núm. 55, págs. 12-19.

FIQUE, L. F. (2008): "La política pública de vivienda en Colombia”, en Revista Bitácora, vol. 13, núm. 2, págs. 73-89.

GARCIA, E. (2014): "Evaluación de las políticas públicas para el desarrollo: efectividad, eficiencia y rentabilidad", en ICE, Revista de Economía, núm. 880, Globalización y Desarrollo, págs. 151-163.

GOBIERNO DE ESPAÑA (2010): Fundamentos de evaluación de políticas públicas, Ministerio de Política Territorial y Administración Pública, Agencia Estatal de Evaluación de las Políticas Públicas y la Calidad de los Servicios, Primera Edición.

henAO, L. F., HERRERA, G., TRUJILlo, N. A. \& CÁRDENAS, A. (2014): Colombia: Cien años de políticas habitacionales. Bogotá, Colombia: Ministerio de Vivienda, Ciudad y Territorio para el Séptimo Foro Mundial.

KELLETT, P., TORO, A. \& HARAMOTO, E. (1994); "Cambios iniciados por los habitantes y transformaciones en la vivienda social: teoría y práctica en el contexto chileno", en Boletín INVI, núm. 21, págs. 3-14.

LOPEZ, H. F. (2011): "Lauchlin Currie y el desarrollo Colombiano", en Criterio Libre, vol. 9, núm. 14, págs. 21-42.

MINISTERIO PARA LAS ADMINISTRACIONES PÚBLICAS (1992): Análisis de políticas públicas y eficacia de la administración, Madrid: Secretaría General Técnica Instituto Nacional de Administración Pública.

MONTENEGRO, A. (2012): "Lauchlin Currie: Desarrollo y crecimiento económico", en Revista de Economía Institucional, vol. 14, núm. 27, págs. 81-97.

NINA, E. (2008): "Modelos de evaluación de políticas y programas sociales en Colombia", en Papel Político, núm. 13, págs. 449-471.

PECHA, C. (2011): Programa de vivienda de interés social de Colombia: Una Evaluación. New York, Washintong, D. C.: Banco Interamericano de Desarrollo.

SALAZAR, C. (2009): "La evaluación y el análisis de políticas públicas", en Revista Opera, núm. 9, págs. 23-51. Recuperado de $h$ ttp://www.redalyc.org/articulo.oa?id=67515007003.

SECRETARÍA DISTRITAL DE HÁBITAT (2015): Política Distrital del Hábitat. Bogotá: Subdirección de Información Sectorial.

VÉLEZ, L. (2013): "Lauchlin Currie: el maestro de los economistas colombianos", en Lecturas de Economía, Universidad de Antioquia, núm. 79, págs. 233-239. DOI: https://doi.org/10.17533/udea.le.n79a8.

ZULUAGA, B. (2003): "Lauchlin Currie: Desarrollo, pobreza y desigualdad del ingreso", en Estudios Gerenciales, núm. 87 , págs. $55-65$. 